\title{
Identification of Coupling Mechanisms between Ultraintense Laser Light and Dense Plasmas
}

\author{
L. Chopineau, ${ }^{1}$ A. Leblanc, ${ }^{1}$ G. Blaclard, ${ }^{1,2}$ A. Denoeud, ${ }^{1}$ M. Thévenet, ${ }^{2}$ J-L. Vay, ${ }^{2}$ G. Bonnaud, ${ }^{1}$ \\ Ph. Martin, ${ }^{1}$ H. Vincenti, ${ }^{1, *}$ and F. Quéré ${ }^{1, \dagger}$ \\ ${ }^{1}$ LIDYL, CEA, CNRS, Université Paris-Saclay, CEA Saclay, 91191 Gif-sur-Yvette, France \\ ${ }^{2}$ Lawrence Berkeley National Laboratory, Berkeley, California 94720, USA
}

(Received 5 September 2018; revised manuscript received 21 December 2018; published 21 March 2019)

\begin{abstract}
The interaction of intense laser beams with plasmas created on solid targets involves a rich nonlinear physics. Because such dense plasmas are reflective for laser light, the coupling with the incident beam occurs within a thin layer at the interface between plasma and vacuum. One of the main paradigms used to understand this coupling, known as the Brunel mechanism, is expected to be valid only for very steep plasma surfaces. Despite innumerable studies, its validity range remains uncertain, and the physics involved for smoother plasma-vacuum interfaces is unclear, especially for ultrahigh laser intensities. We report the first comprehensive experimental and numerical study of the laser-plasma coupling mechanisms as a function of the plasma interface steepness, in the relativistic interaction regime. Our results reveal a clear transition from the temporally periodic Brunel mechanism to a chaotic dynamic associated to stochastic heating. By revealing the key signatures of these two distinct regimes on experimental observables, we provide an important landmark for the interpretation of future experiments.
\end{abstract}

DOI: 10.1103/PhysRevX.9.011050

Subject Areas: Nonlinear Dynamics, Plasma Physics

\section{INTRODUCTION}

High-density plasmas can be created by focusing intense laser pulses on initially solid targets. The interaction of such plasmas with laser light, investigated for several decades, involves a rich nonlinear physics which is not only of fundamental interest but also of high relevance for a wide range of applications over a large interval of laser intensities, spanning thermonuclear fusion [1], laboratory astrophysics [2], or laser-driven particle acceleration [3,4]. For most, if not all, applications, depositing laser energy into the plasma is essential. Because of their high density, largely in excess of the so-called critical density where the local electron plasma frequency equals the laser frequency, these plasmas, however, tend to reflect a large fraction of the laser light. The actual coupling with the incident light field can only occur either in the undercritical part of the density gradient at the plasma-vacuum interface, where the laser wave propagates, or within the skin depth of the overcritical plasma, where the laser wave is evanescent.

\footnotetext{
*henri.vincenti@cea.fr

†fabien.quere@cea.fr
}

Published by the American Physical Society under the terms of the Creative Commons Attribution 4.0 International license. Further distribution of this work must maintain attribution to the author(s) and the published article's title, journal citation, and DOI.
At such plasma densities, physicists initially anticipated the main mechanism of energy deposition to be collisional absorption [5]: electron-ion collisions disrupt the regular quivering motion of the plasma electrons in the light field, statistically leading to a net kinetic energy gain from the laser field. Soon after the invention of lasers, experimental studies on the feasibility of laser-driven thermonuclear fusion, however, revealed the importance of noncollisional light absorption mechanisms, coming into play for moderate laser intensities $\left(I \lambda^{2} \gtrsim 10^{13} \mathrm{~W} \mu \mathrm{m}^{2} / \mathrm{cm}^{2}\right)$ [6]. For interactions at the surface of dense plasmas, these processes are expected to be most relevant when the laser beam impinges the target at oblique incidence (angle of incidence $\left.\theta_{i} \neq 0\right)$ and in $p$ polarization, such that an electric field component efficiently drives electron motion along the normal to the target surface. Among these so-called "anomalous absorption" mechanisms, the resonant excitation and subsequent damping of collective electronic plasma waves at the critical plasma density has been the first key process to be identified both theoretically and experimentally, and is commonly known as resonant absorption [7-10].

Since the coupling with laser light occurs at the interface of the plasma with vacuum, the characteristic spatial length $L$ of the plasma density gradient across this interface is a crucial parameter. This density gradient is generally not steplike, due to the unavoidable expansion of the plasma into vacuum, either during the main laser pulse driving the interaction or even before this pulse when laser prepulses 
are present (either accidentally or voluntarily introduced). In an influential Letter from 1987, Brunel predicted a transition from resonant absorption to a new coupling mechanism, which he ironically called "not-so-resonant, resonant absorption" [11], depending on the value of $L$. He anticipated this mechanism to come into play when the laser intensity becomes so strong that the quivering amplitude of the plasma electrons in the field along the target normal gets larger than $L$, such that the plasmavacuum interface can be modeled as steplike.

This simple and intuitive mechanism, now known as Brunel absorption or vacuum heating, is qualitatively analogous to the intensively studied three-step model of atomic and molecular strong-field physics, where an intense laser field $\left(I \lambda^{2} \gtrsim 10^{13} \mathrm{~W} \mu \mathrm{m}^{2} / \mathrm{cm}^{2}\right)$ drives the recollision of ionized electrons with their parent ions [12]. Here, in each optical laser cycle, electrons at the target surface are dragged out of the plasma into vacuum when the laser $E$-field component normal to the target is directed towards the plasma (see simulation results in Fig. 1). Later in the cycle, when this driving field changes sign, some of these expelled electrons are pushed back toward the "parent plasma": as they penetrate into this dense plasma, they escape the influence of the laser field due to plasma screening and propagate ballistically into the target (red trajectory in Fig. 1). The initial model by Brunel for a steplike surface of a perfectly conducting plasma focused on this returning electron population. However, numerical simulations show that for smoother interfaces and/or higher laser intensities,

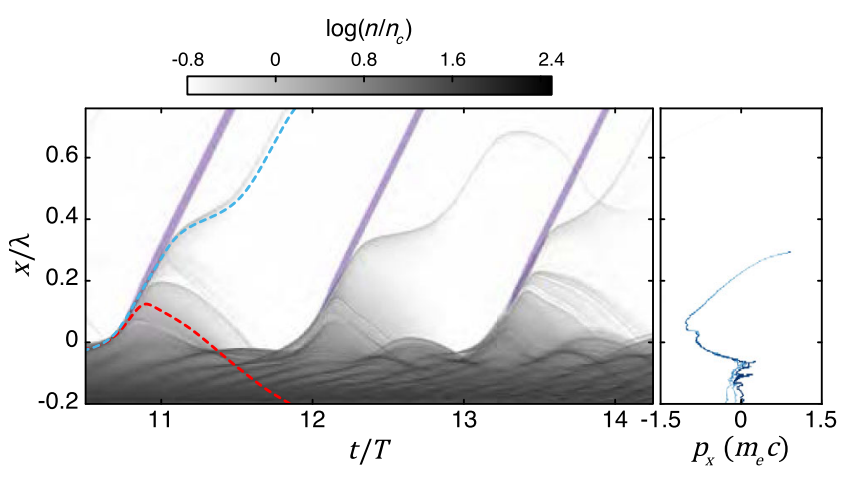

FIG. 1. Temporal dynamics of a dense plasma exposed to an ultraintense laser field in the Brunel regime. This graph displays results from a particle-in-cell simulation performed for $a_{0}=2$ $\left(I=8.5 \times 10^{18} \mathrm{~W} / \mathrm{cm}^{2}, \lambda=800 \mathrm{~nm}\right), \theta_{i}=55^{\circ}$, and a density gradient scale length $L=\lambda / 10$ (with $\lambda$ the laser wavelength). The gray scale color map shows the temporal evolution, during three laser optical periods, of the plasma electron density around the target surface, while the purple color scale shows the attosecond light pulses emitted by this plasma (harmonics 8-15). Two representative trajectories for the particles forming the expelled electron beam (blue) and the "recolliding" electron flux (red) are also displayed. The right-hand panel shows a typical distribution of electrons in the $x-p_{x}$ phase space ( $x$ spatial coordinate along the target normal) at time $t / T=12.7$. another fraction of the electrons escapes into vacuum (blue trajectory in Fig. 1), typically in the form of periodic attosecond bunches. In both cases, the fast electrons resulting from this sub-optical-cycle dynamics carry away energy acquired from the laser: for convenience, the terms "Brunel electrons" or "Brunel absorption" used in this paper will encompass these two populations.

A few years later, with the development of high-power femtosecond lasers, the Brunel mechanism appeared as an ideal toy model to understand the interaction of dense plasmas with these ultrashort pulses. First, their ultrahigh intensities result in large electron quivering amplitudes. Second, these pulses are so short that plasma expansion during the interaction is very limited, potentially leading to sharp density gradients at the plasma surface, i.e., small values of $L$. However, from an experimental point of view, reaching this regime turned out to be much more challenging than expected. The key difficulty arose from the unavoidable light pedestal ahead of ultrashort laser pulses [13]: this pedestal, if too intense, leads to the premature creation and expansion of the plasma, and thus to long and largely uncontrolled density gradients at the plasmavacuum interface when the main laser pulse hits the target, making the Brunel regime inaccessible. More generally, this major issue has considerably complicated the interpretation of most early experiments on the interaction of intense ultrashort lasers with dense plasmas.

It took more than an additional decade to find methods to efficiently reduce the light pedestal accompanying ultrashort laser pulses [14-17], and thus obtain temporal contrasts that at last made extremely sharp plasma surfaces accessible and compatible with ultrahigh laser intensities $[18,19]$. Nowadays, the Brunel mechanism is most likely at play in experiments performed on solid targets with ultraintense laser pulses of suitably high contrast. Yet, direct experimental evidence is still elusive, and its range of validity is not precisely known so far. Furthermore, following the historical development of this topic, the "common wisdom" tends to be that when $L$ is increased, a transition from Brunel to resonant absorption should at some point occur [20-22], but no clear experimental evidence of this transition has been reported yet.

A broad range of topical experiments are now being performed worldwide on the interaction of ultraintense laser pulses $\left(I \lambda^{2}>10^{18} \mathrm{~W} \mu \mathrm{m}^{2} / \mathrm{cm}^{2}\right)$ with dense plasmas, driven by applications such as laser-driven ion [3,4,23,24] and electron acceleration [25-32], or the generation of intense harmonics and/or attosecond light pulses [22,33]. Clearly identifying the laser-plasma coupling mechanisms at play in this interaction regime, and determining the range of physical parameters where they are relevant, is essential for the proper understanding of such experiments. This is what we achieve in this article, by focusing ultraintense femtosecond laser pulses on a dense plasma with a sharp, controlled and measured density gradient scale length $L$, 
which we systematically vary from $L \ll \lambda$ to $L \approx \lambda$ (with $\lambda$ the laser wavelength). We show how performing and correlating measurements of the high-order harmonics and relativistic electrons emerging from the target provide clear signatures of these couplings mechanisms, and relate these observations to the underlying physics through an advanced analysis of 2D and 3D particle-in-cell (PIC) simulations, solving the coupled Vlasov-Maxwell equation system.

This comprehensive study shows that the Brunel mechanism is indeed the relevant physical process for sharp enough plasma-vacuum interfaces. As expected, a transition occurs to a different mechanism when the density gradient scale length $L$ is increased. Measurements of this transition as a function of the laser incidence angle provide confirmation of Brunel's transition criteria based on the comparison of the electron quivering amplitude with the typical spatial extent of the interface. However, we establish that in the regime of ultrahigh laser intensities considered here, resonant absorption plays no significant role in the regime of large $L(L \approx \lambda)$. The coupling is rather dominated by another kinetic mechanism, so far known as stochastic heating, in which collective plasma effects play little role: as suggested theoretically in Refs. [34,35], electrons in the underdense part of the density gradient gain energy in the interference pattern resulting from the superposition of the incident laser field with the field reflected by the overdense part of the plasma. It has been established theoretically that at the laser intensities considered here, electron dynamics in such an interference pattern is not integrable, gets chaotic and can lead to high energy transfer from the laser wave to the electron population [36].

In this paper, the amplitude of the incident laser field, which determines the intensity on target, is characterized by the dimensionless potential vector at the peak of the pulse, $a_{0}=e E_{0} / m c \omega=\lambda[\mu \mathrm{m}]\left(I\left[\mathrm{~W} / \mathrm{cm}^{2}\right] / 1.37 \times 10^{18}\right)^{1 / 2}$, with $c$ the speed of light, $e$ the elementary charge, $m$ the electron mass, $\omega$ the laser frequency, and $E_{0}$ the amplitude of the laser electric field. All experiments and simulations presented here have been performed with $a_{0}>1$, which corresponds to the interaction regime where relativistic effects play an important role on electron motion. We define $n_{c}$ as the critical plasma density associated to the laser angular frequency $\omega\left(n_{c}=m \epsilon_{0} \omega^{2} / e^{2}=1.74 \times 10^{21} \mathrm{~cm}^{-3}\right.$ for $\lambda=800 \mathrm{~nm}$ laser light). In the following, the incidence angle of the laser beam on target is defined with respect to the target normal, and is expressed in degrees. In contrast, the angular directions of photon and particle emissions from the target are defined with respect to the specular direction, and is expressed in milliradians (mrad) owing to their much smaller values.

\section{DESCRIPTION OF THE EXPERIMENT}

A sketch of the experiment is presented in the upper part of Fig. 2. A high-power femtosecond laser beam is focused on an optically polished silica target, which it fully ionizes on a thin surface layer [22], thus producing a dense plasma (maximum plasma density $n_{0} \simeq 6 \times 10^{23} \mathrm{~cm}^{-3}$, i.e., $400 n_{c}$ for $800 \mathrm{~nm}$ light).

We use the UHI100 laser at CEA Saclay, a commercial system delivering 20-25 fs pulses with a peak power of 100 TW. After correction of its wave front by an adaptive optical system, the beam is focused by an off-axis parabolic mirror with an aperture of $f / 6$, leading to a focal spot of $5 \mu \mathrm{m}$ diameter (FWHM in intensity) and to an estimated peak intensity of $2 \times 10^{19} \mathrm{~W} / \mathrm{cm}^{2}\left(a_{0} \approx 3.5\right)$ on target. By default, the laser beam is $p$ polarized on target, but the polarization can be switched to $s$ by inserting a thin zeroorder mica half-wave plate in the beam.

The first key aspect of the experiment is that it was carried out with a high degree of control and an accurate knowledge of the plasma density gradient scale length $L$ at the target surface, which is a prerequisite for the study of the laser-plasma coupling mechanisms. This implies the use of laser pulses of very high temporal contrast, so that premature creation of the plasma on target is avoided: this is achieved thanks to a double plasma mirror system placed before the main experimental chamber [18], which increases the contrast by about 4 decades, thus reaching $\gtrsim 10^{13}$ for time delays $\gtrsim 100$ ps before the main pulse. This ultrahigh temporal contrast is of paramount importance for all experiments presented here. Starting from the very steep density gradient allowed by this ultrahigh temporal contrast, $L$ is then varied in a controlled way, thanks to the introduction of a "weak" prepulse (fluence $\approx 1 \mathrm{~kJ} / \mathrm{cm}^{2}$ ) at an adjustable delay $\tau$ before the main pulse $(0 \leq \tau \leq 15 \mathrm{ps})$, produced from an edge of the main beam using the optical layout described in Ref. [37]. This prepulse is strong enough to ionize the target and initiate plasma expansion, at a typical velocity in the $40-60 \mathrm{~nm} / \mathrm{ps}$ range. For all experimental conditions considered herein, and in particular for all incidence angles, we systematically measured $L(\tau)$ using the recently introduced technique of spatial domain interferometry (SDI) [38]; see Supplemental Material [39].

The second key aspect of the experiment is the combination of diagnostics that were implemented to study the interaction. We concentrated on two types of observables: the relativistic electron beam emitted by the target towards vacuum and the beam of high-order harmonics generated around the specular reflection direction.

Two diagnostics were used for the electron beam. First, a LANEX screen, placed behind a $13-\mu \mathrm{m}$-thick aluminum foil (to eliminate laser light and its harmonics) and a 2-mmthick glass plate (to filter out low-energy electrons), and the fluorescence of which was imaged on a CCD camera, provided the spatial profile of the emission of electrons with energies higher than $1 \mathrm{MeV}$, at a distance of $\approx 10 \mathrm{~cm}$ from the target [left-hand images in Figs. 2(b) and 2(c)]. Second, we designed a new type of magnetic spectrometer for relativistic electrons (see Supplemental Material [39]), 


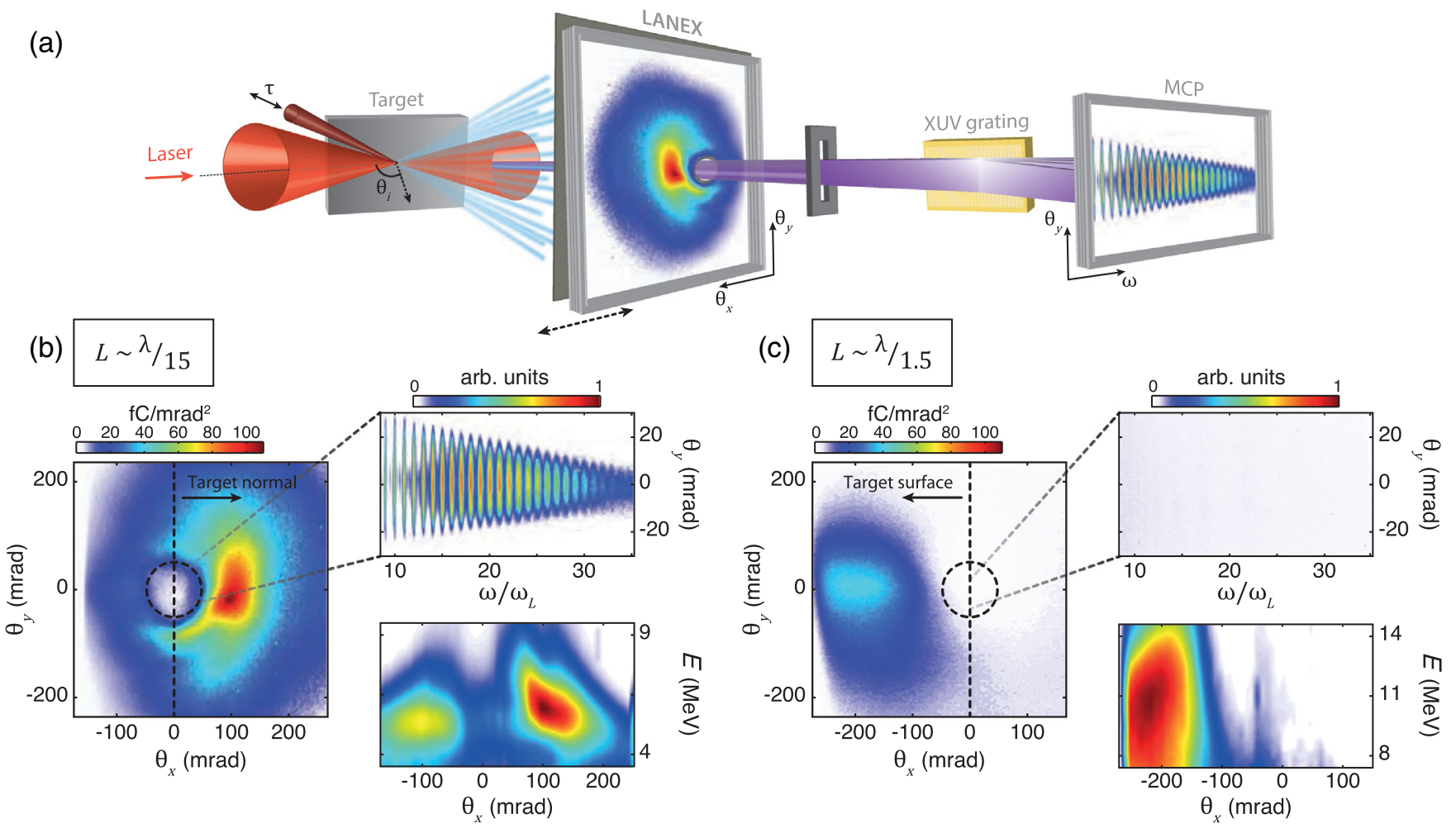

FIG. 2. Principle of the experiment and main experimental findings. (a) Sketch of the experiment. The target is impinged by a controlled prepulse, followed by the main pulse after an adjustable delay $\tau$. Two of the main diagnostics are displayed: the LANEX screen for the measurement of the spatial profile of the high-energy electron beam and the angularly resolved XUV spectrometer, equiped with a micro channel plate (MCP) detector. These two diagnostics can be used either separately or simultaneously when small holes are made in the electron detection assembly, as shown in the figure. They can also be replaced by an angularly resolved electron spectrometer. The main experimental findings for a $p$ polarized laser field are summarized in (b) and (c): left-hand images, angular emission pattern of relativistic electrons; bottom right-hand images, angularly resolved energy spectrum of electrons in the incidence plane $\left(\theta_{y}=0\right)$; top right-hand images, angularly resolved harmonic spectrum. In panel (b), the experimental parameters are $\theta_{i}=55^{\circ}$, $a_{0}=3.5, \tau=1 \mathrm{ps}$, leading to a gradient scale length $L=\lambda / 15$. In panel (c), the experimental parameters are the same, except for $\tau=10 \mathrm{ps}$, leading to a gradient scale length $L=\lambda / 1.5$. These illustrate the major changes occurring on these three observables as the density gradient scale length is increased from $L \ll \lambda$ to $L \sim \lambda$, which constitute signatures of the different underlying laser-plasma coupling mechanisms. In panels (b) and (c), the circular dashed lines correspond to the angular extent of the reflected laser beam.

which provided, for every laser shot, the angularly resolved kinetic energy spectrum of electrons, in the incidence plane (i.e., for $\left.\theta_{y}=0\right)$. This $\left(\theta_{x}, E\right)$ distribution, with $\theta_{x}$ the angle in the plane of incidence and $E$ the electron kinetic energy, was measured with a very large angular acceptance of $\Delta \theta_{x}=500 \mathrm{mrad}$ around the specular reflection direction $\left(\theta_{x}=0\right)$ [bottom right-hand images in Figs. 2(b) and 2(c)].

The harmonic beam was characterized using an angularly resolved extreme ultraviolet (XUV) spectrometer [37], with an angular acceptance of $200 \mathrm{mrad}$ around the specular direction [top right-hand images in Figs. 2(b) and 2(c)]. The harmonic spectrum and the electron beam spatial profile were initially measured on the same laser shots, thanks to small holes in the aluminum foil, glass plate, and LANEX screen that let the harmonic beam go through (Fig. 2). However, we observed an excellent shot-to-shot reproducibility of the experimental results, so that these multiple diagnostics were finally implemented on different laser shots performed under identical interaction conditions.

A simple additional diagnostic, implemented on separate laser shots, consisted of measuring the spatial profile of the laser beam reflected by the target, by inserting a frosted glass plate in this beam $20-30 \mathrm{~cm}$ after the target, and measuring the image of the laser light scattered by this plate on a camera placed behind a bandpass filter centered on the fundamental laser frequency. This can be exploited to determine the plasma reflectivity for the incident laser beam.

\section{EXPERIMENTAL RESULTS}

The lower part of Fig. 2 summarizes the main findings of the experiment for a $p$ polarization of the incident laser, by presenting the electron beam angular profiles and $\left(\theta_{x}, E\right)$ distributions, as well as the angularly resolved harmonic 

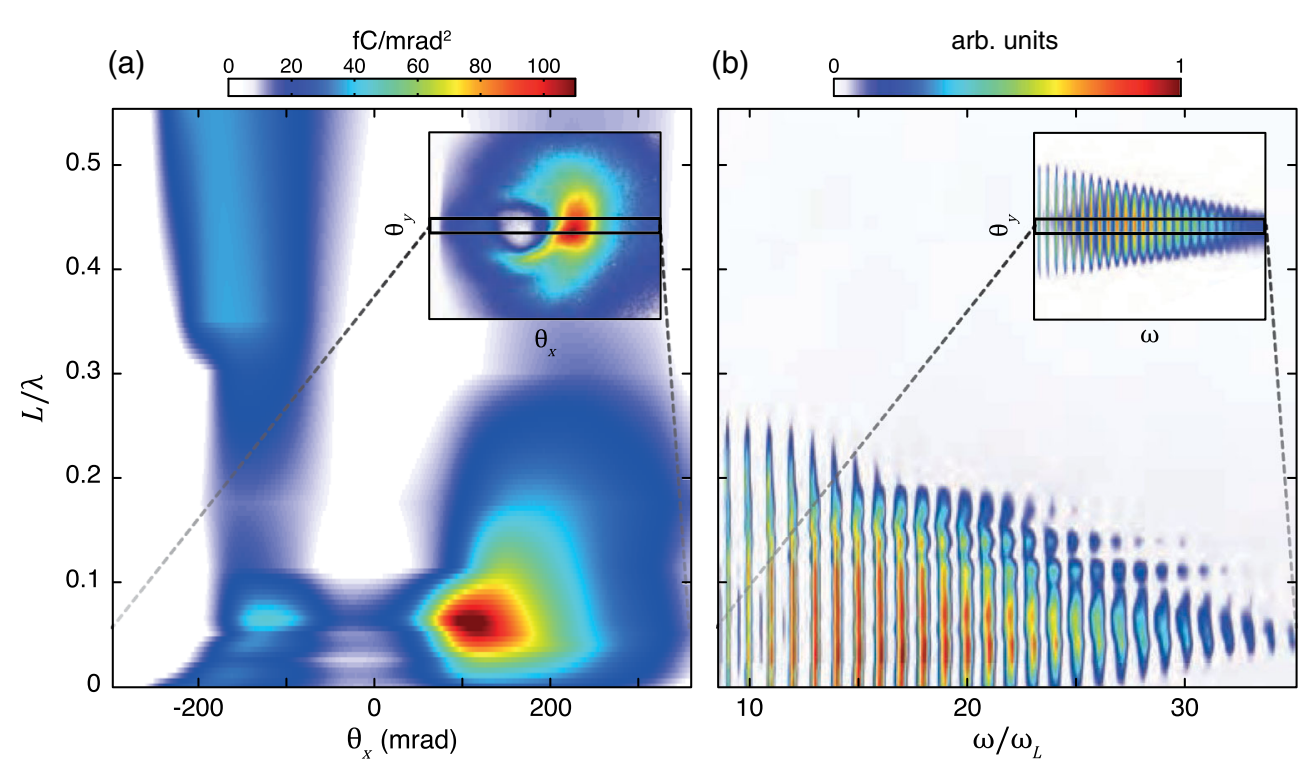

(c)

FIG. 3. Evolution of the experimental observables with the density gradient scale length. The angular profile of the relativistic electron beam in the incidence plane (a) and the emitted harmonic spectrum (b) are plotted as a function of $L$, for a $p$-polarized laser field. The experimental parameters are the same as in Fig. 2. The insets show how these quantities are related to the images of Fig. 2 (note that these insets do not hide any relevant signal). Panel (c) shows different curves derived from these datasets: the harmonic signal integrated from the 20th to the 25 th order ( $\left(-250 \leq \theta_{x} \leq-150 \mathrm{mrad}\right.$, signal $\left.S_{e 2}\right)$ of the specular direction, are plotted as a function of $L$. These curves show the transition between the two regimes highlighted in Fig. 2, and reveal the quantitative correlation between the harmonic and relativistic electron signals for short-gradient scale lengths.

spectra, measured for two different density gradient scale lengths $L, L_{1} \ll \lambda$ and $L_{2} \approx \lambda$ : as $L$ is increased from $L_{1}$ to $L_{2}$, the measured signals for all these observables radically change. Three main differences are observed: (i) when $L \ll \lambda$, the electron emission is predominantly peaked at $\theta_{x} \approx 100 \mathrm{mrad}$, i.e., close to the direction of laser specular reflection $\left(\theta_{x}=0 \mathrm{mrad}\right)$, with a slight shift towards the target normal. As $L$ is increased, it then switches to $\theta_{x} \approx-200 \mathrm{mrad}$, a direction between specular direction and the tangent to the target surface $\left(\theta_{x}=-600 \mathrm{mrad}\right)$, and simultaneously slightly broadens angularly. (ii) Electrons reach energies about 2 times higher for large $L$ (spectral peak around $10 \mathrm{MeV})$, with a $\left(\theta_{x}, E\right)$ distribution that significantly changes. In the short-gradient regime, a clear correlation is observed between emission angle $\theta_{x}$ and electron energy $E$, especially in the most intense part of the distribution $\left(0 \leq \theta_{x} \leq 200 \mathrm{mrad}\right)$ : the electron energy increases as one gets closer to the specular direction. In contrast, in the long-gradient regime, the electron spectrum hardly varies angularly; i.e., no significant correlation is observed on this $\left(\theta_{x}, E\right)$ distribution. (iii) Harmonic emission is clearly observed for small $L$, but it drops below the experimental detection threshold for large $L$.

The details of the transition between these two regimes are presented in Fig. 3, which displays the evolution with $L$ of the electron beam angular profile in the incidence plane, and of the harmonic spectrum. The most important point is the quantitative correlation, observed at short gradients, between the emission of relativistic electrons and the harmonic signal [see curves in Fig. 3(c)]. As $L$ is gradually increased, the electron signal around $\theta_{x}=100 \mathrm{mrad}$ and the harmonic signal reach a common optimum around $L=$ $\lambda / 15$, and then both quickly decrease. The electron signal on the other side of the specular direction then grows, but is not associated with any harmonic signal. The transition between these two regimes occurs around $L \approx \lambda / 5$.

Another important difference between these two interaction regimes is the dependence of the observables on laser polarization direction, illustrated in Fig. 4. In the short-gradient regime, the electron signal is totally suppressed when the polarization is switched from $p$ to $s$, as is also the case for the harmonic signal (not shown). By contrast, for longer gradients, the electron signal is still observed for $s$ polarization, although it gets about 5 times weaker.

These observations on the electron and harmonic beams clearly point to a complete change in the coupling mechanism between the laser field and the plasma, which we analyze in the rest of this article. In the following, we refer to these two interaction regimes as the short-gradient and long-gradient regimes for convenience.

This transition also has consequences on even simpler observables: Fig. 5 (left-hand panels) displays the spatial intensity profiles of the reflected laser beam, measured on a scattering screen in these two distinct coupling regimes. In the short-gradient regime, Fig. 5(a), a smooth beam 

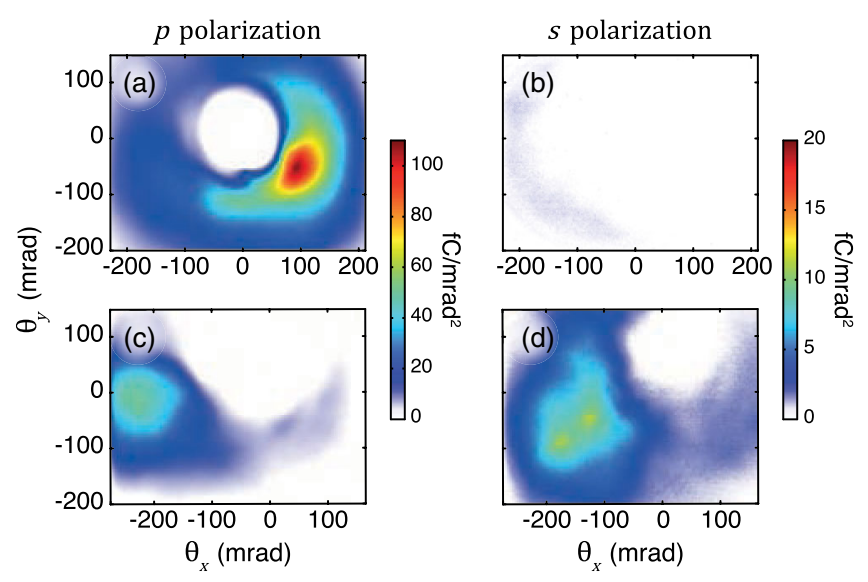

FIG. 4. Effect of the laser polarization direction on relativistic electron emission. Panels (a) and (b) show the angular profiles of the relativistic electron emission for a gradient scale length $L_{1}=\lambda / 15$, respectively for $p$ and $s$ polarizations of the incident laser. In both cases, the laser incidence angle is $\theta_{i}=55^{\circ}$. Panels (c) and (d) show these electron angular distributions, now measured for a longer gradient $L_{2}=\lambda / 1.5$, i.e., beyond the transition observed in Fig. 3. Note the different color scales used in (a) and (c) versus (b) and (d).

is observed, which is almost unaltered compared to the incident laser beam: this is the so-called plasma mirror regime [19], where the plasma acts as a usual high-quality mirror, specularly reflecting the fundamental frequency, despite the ultrahigh intensity on target. By contrast, in the long-gradient regime Fig. 5(b), the beam profile is strongly perturbed and starts exhibiting spatial structures that were not present on the incident beam. The term plasma mirror is thus no longer appropriate to this regime, although the laser field still interacts with a dense-and hence reflectiveplasma. Experimentally, the spatial profile of the reflected laser beam might thus also be used as an alternative and very simple signature of the transition in the laser-plasma interaction. By spatially integrating these images, the variation of the plasma reflectivity at the fundamental laser frequency as a function of $L$ can be determined, and is displayed in the right-hand panel of Fig. 5, for both $p$ and $s$ polarizations of the incident laser field.

All these measurements have been repeated over four different experimental campaigns on the UHI100 experimental facility over the past four years, and all effects described above have been observed to be very reproducible and robust.

\section{3D PARTICLE-IN-CELL SIMULATIONS}

To interpret these experimental observations, we will now turn to particle-in-cell (PIC) simulations of the laserplasma interaction. This requires ensuring that (i) these PIC simulations are performed in the actual physical conditions of the experiment and (ii) they are reliable and properly reproduce the key experimental findings. To check these
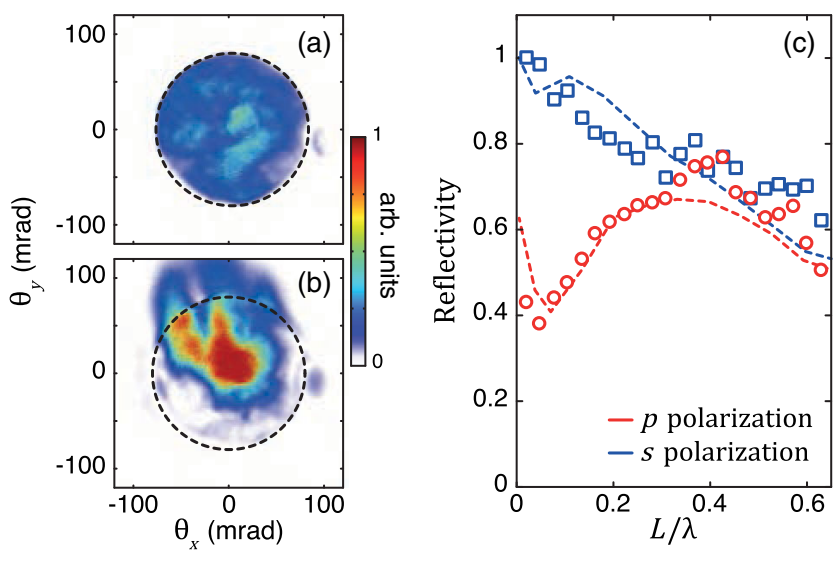

FIG. 5. Reflected fundamental beam and evolution of the plasma reflectivity. Using a scattering screen, the spatial intensity profile of the laser beam reflected by the target can be measured. The two panels (a) and (b) show typical beam profiles respectively obtained in the short (upper image, $L_{1}=\lambda / 15$ ) and long (lower image, $L_{2}=\lambda / 1.5$ ) density gradient regimes, for $p$ polarization of the incident laser. In all cases, the laser incidence angle is $\theta_{i}=55^{\circ}$. The black dashed circles indicate the initial divergence of the top-hat laser beam, before its interaction with the target. From the spatial integration of these images, the reflectivity of the plasma for the fundamental laser frequency can be determined, and is plotted in (c) as a function of $L$ for both $s$ and $p$ polarizations (squares and circles). The lines show the corresponding results of 2D particle-in-cell simulations (see Sec. V D).

two critical points, we first carry out full 3D simulations of the interaction, so that we can directly confront the numerical and experimental results-especially the full angular pattern of the electron emission, which is accessible only by $3 \mathrm{D}$ simulations.

All simulations reported herein have been performed using the recently developed WARP+PXR code [40-44]. The specificity of this code is the use of a massively parallel high-order spectral solver for Maxwell's equations [45], which greatly reduces numerical dispersion of electromagnetic waves as well as numerical noise. This ensures convergence of the simulations for larger spatial and temporal mesh steps than in most other PIC codes, and thus makes physically realistic and reliable $3 \mathrm{D}$ simulations of the interaction with dense plasmas computationally tractable [45,46]. Each 3D simulation reported here required $6.3 \times 10^{6}$ computation hours on a massively parallel machine $[47,48]$. More detailed information on the numerical parameters of these simulations is provided in the Supplemental Material [39].

We performed two 3D simulations, for the same physical conditions as in the experiments of Figs. 2(b) and 2(c), corresponding to fixed laser parameters but different density gradient scale lengths, $L_{1} \ll \lambda$ and $L_{2} \approx \lambda$. From these simulations, we extracted the exact same observables as those measured in the experiment; the calculated angular profiles and angle-energy distributions of the emitted 

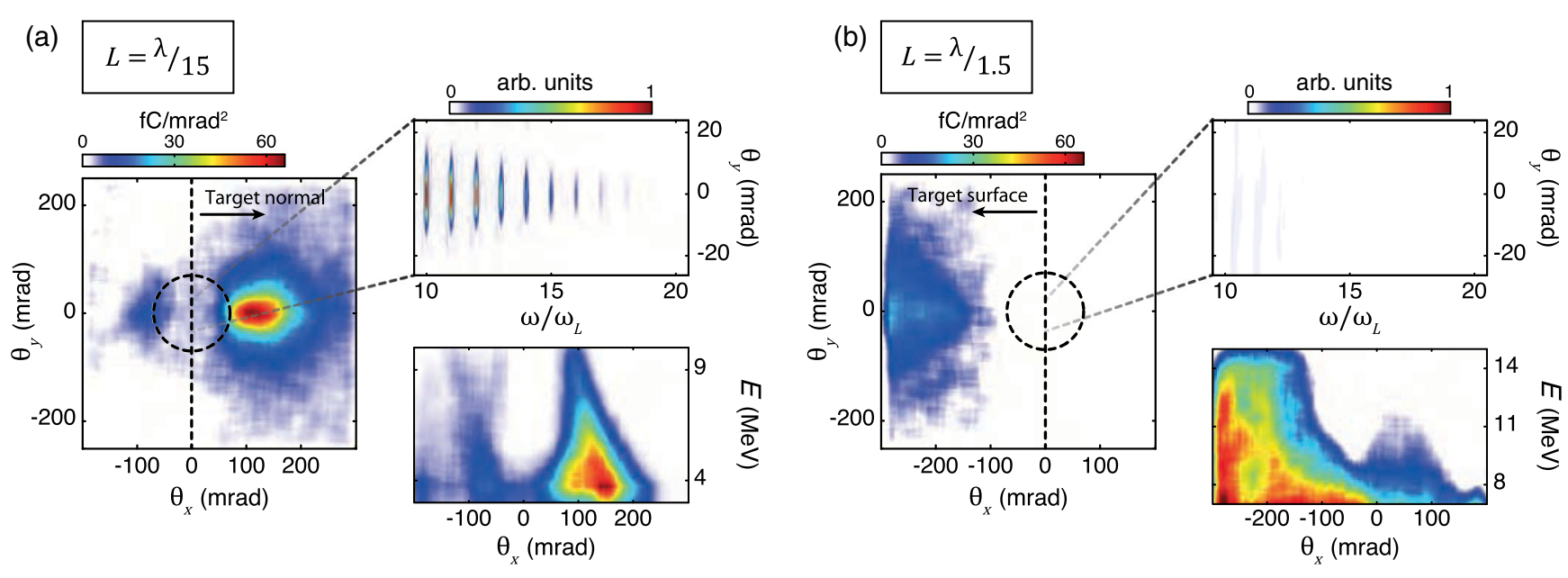

FIG. 6. 3D PIC simulations of the laser-plasma interaction for two different density gradient scale lengths, $L$. The physical conditions of these simulations are matched to the estimated experimental conditions of the shots shown in the lower part of Fig. 2: panel (a) corresponds to a density gradient $L_{1}=\lambda / 15$ and panel (b) to $L_{2}=\lambda / 1.5$, while all other physical parameters remain the same. From these simulations, we extract the same observables as those measured in the experiment: the angular profile of the high-energy electron beam expelled in vacuum (left-hand image in each panel), the $\left(\theta_{x}, E\right)$ distribution of these electrons (bottom right-hand image in each panel), and the angularly resolved harmonic spectrum (top right-hand image in each panel). The key features are the same as those observed on the experimental results (compare with the lower panels of Fig. 2).

electron beam, as well as the angularly resolved harmonic spectra, are displayed in Fig. 6. Comparison with the lower panels of Fig. 2 shows that these simulations very well reproduce the two distinct interaction regimes observed experimentally for all these observables.

These 3D benchmark simulations clearly demonstrate both the reliability of the PIC simulations as well as our excellent control of the interaction conditions in the experiments, which ensures a relevant choice of the physical parameters used in the theoretical study. In the next section, we further exploit simulations performed with the WARP + PXR code to get detailed insight into the physical processes underlying these distinct interaction regimes. To this end, we rely on more tractable $2 \mathrm{D}$ simulations [49]. Detailed information on the numerical parameters of these simulations is also provided in the Supplemental Material [39].

\section{PHYSICAL ANALYSIS AND DISCUSSION}

The starting point for our analysis of the experimental results is the joint measurement of the relativistic electron and harmonic signals, which provides information on the temporal structure of the electron emission by the plasma (Sec. VA). We then discuss the spatial structure of the observed electron beams (Sec. V B). In order to understand the electron heating and ejection mechanism in the longgradient regime, we turn to a detailed analysis of PIC simulations, presented in Sec. VC. With the physical insight provided by this analysis, we finally discuss the influence of the laser polarization (Fig. 4) and the evolution with $L$ of the plasma reflectivity at the fundamental laser frequency (Fig. 5) in Sec. V D.

\section{A. Temporal structure of the electron emission}

The clear correlation observed between the high-energy electron signal and the harmonic signal for short gradients [Fig. 3(c)] suggests that in this regime, the relativistic electrons are involved in the harmonic emission. Then, the fact that a highly contrasted harmonic comb is produced would be an indication that this electron emission is periodic in time, being locked to the driving laser field.

To support this tentative interpretation, we consider the PIC simulations of Figs. 1 and 7(b): they show that temporal periodicity is indeed a characteristic of the Brunel mechanism. Electron emission occurs in the form of bunches that are initially extremely short (in the attosecond range), emitted once every optical period. For $a_{0}>1$, these electrons reach relativistic velocities when they escape the plasma, and thus induce a Doppler effect on the reflected laser field: this results in the generation of a train of attosecond light pulses, spaced by one laser period, which are clearly observed in Figs. 1 and 7. This wellidentified process is known as the relativistic oscillating mirror (ROM) effect [19,50-58]. In simulations, the resulting periodic light emission has a spectrum consisting of a comb of high-order harmonics of the laser frequency: this is the origin of the harmonic signal observed in our experiment. The electron-harmonic correlation observed experimentally is therefore a signature of the periodicity of the dynamics of electrons.

As described in Sec. III, the harmonic signal is observed to collapse for longer density gradients $L$. One possible interpretation for this collapse can be that the electron emission ceases to be periodic in time. And, indeed, PIC simulations for longer density gradients [Fig. 7(d)] strikingly 

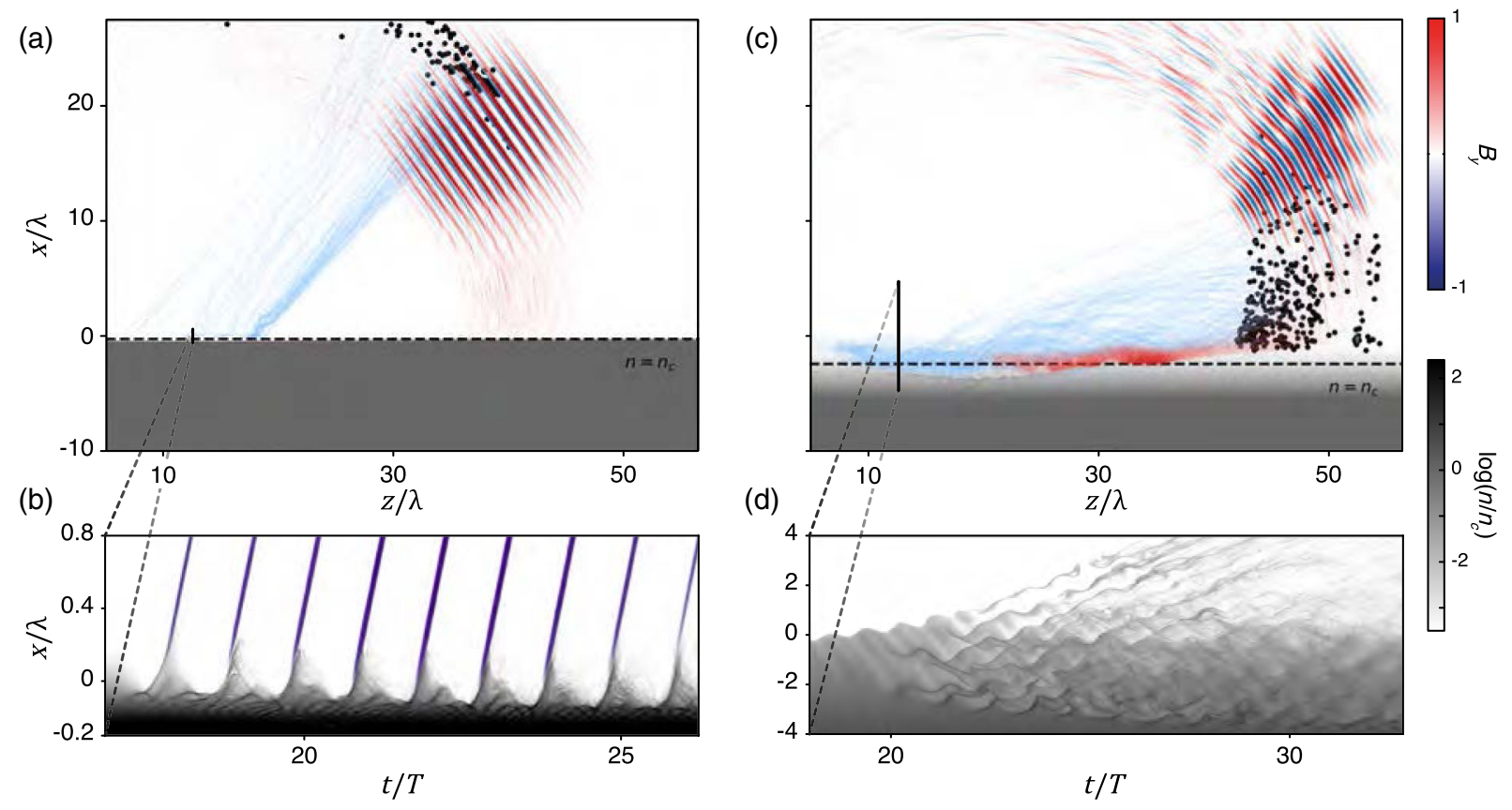

FIG. 7. 2D PIC simulations in the two distinct regimes of laser-plasma coupling. These data are obtained from 2D PIC simulations with different density gradients $L[\lambda / 15$ for (a) and (b), and $\lambda / 1.5$ for (c) and (d)], while all other physical parameters remain the same $\left(a_{0}=3.5, \theta_{i}=55^{\circ}\right.$ ). The two upper panels display the complete trajectories of a selected set of expelled high-energy test electrons (blue lines), together with the $y$ component of the magnetic field (blue to red color map) at a given time after the laser-plasma interaction. The plasma density profile at the end of the interaction is indicated in gray in log scale. The lower panels shows the temporal evolution of the plasma electron density (gray scale color map, in log scale), spatially resolved along the normal to the target surface, at the center of the focal spot. The emitted attosecond pulses are overlaid to this density map in purple. They are clearly visible in (b), but are too weak to be observed in (d).

show that, in contrast to the Brunel mechanism, electron emission is no longer periodic in this regime. The absence of a harmonic signal in conjunction with the relativistic electron emission can thus be considered as a signature of the transition to a new coupling mechanism, associated with the very different plasma temporal dynamics observed in Fig. 7. This mechanism is described in Sec. V C.

\section{B. Spatial structure of the electron emission}

We now discuss the spatial properties of the outgoing electron beams, with the support of the simulation results of Fig. 7. We show that in the short-gradient regime, this structure is mostly determined by the interaction of expelled electrons with the reflected laser field in vacuum (Sec. V B 1), while in the long-gradient regime, it is rather imposed by large quasistatic surface fields that develop in the vicinity of the plasma surface during the interaction (Sec. V B 2).

\section{Short-gradient regime}

In the case of short density gradients, the peculiar angular structure of the electron beam has recently been analyzed experimentally and theoretically in Ref. [32]. In this Brunel regime, electrons are expelled from the plasma as a very laminar beam, with relativistic velocities initially quasiparallel to the direction of specular reflection [Fig. 7(a)]. These relativistic electrons thus copropagate with the intense reflected laser field, with which they interact in vacuum over a distance of the order of the Rayleigh length. This interaction always results in the ejection of electrons out of the laser beam, and therefore forms a hole in the electron beam, centered on the specular direction, as observed in our experiment.

There are two typical scenarios for this ejection, depending on the exact initial conditions with which electrons are expelled from the plasma into vacuum. Some electrons explore multiple optical cycles of the laser field, and thus oscillate in the field and get expelled from the laser focal volume by the so-called ponderomotive effect, isotropically and with a limited energy gain $[59,60]$. They form the ringshaped halo observed on the electron beam. But a large fraction of electrons actually remain around a given phase of the reflected field and rather "surf" a single wave front of the reflected field, thus escaping the laser beam laterally along the laser polarization direction, and forming the bright peak observed next to the specular direction. The side on which this peak forms is determined by the laser phase at which electrons are expelled from the plasma into vacuum: the observation of a peak on one side only of the "ponderomotive hole" (between the specular direction and the target normal) is a clear indication that electrons are 
ejected periodically once every laser period, when the laser field drags them out of the plasma, in the form of suboptical cycle bunches.

This second set of electrons experiences a quasiconstant $E$ field from the laser in vacuum until they escape the focal volume, leading to a greater energy gain than in ponderomotive scattering: this "vacuum laser acceleration" (VLA) process accounts for the observed asymmetry of the $\left(\theta_{x}, E\right)$ distribution [see Fig. 2(b)], where higher energies are observed on one side of the hole (mostly VLA electrons) than on the other (ponderomotive electrons), as well as for the angle-energy correlations on this distribution [61]. An important consequence is that the Doppler up-shift factor induced by outgoing electrons on the reflected field, which leads to the generation of high-order harmonic (ROM mechanism), cannot be directly deduced from the electron spectra measured experimentally, since electrons keep gaining energy after they escaped the target and emitted high-order harmonics, and before being detected. For instance, simulations show that in the present experiment, the electron Lorentz factor typically varies from $\gamma \approx 2-3$ as they are ejected from the plasma and emit harmonics, to $\gamma \gtrsim 15$ after their interaction with the reflected laser field [32], when they are detected.

\section{Long-gradient regime}

The spatial properties of the electron beam observed in the long-gradient regime described here have not been explained in detail yet, to the best of our knowledge. The electron trajectories displayed in Fig. 7 show that the conditions of electron ejection from the plasma are already very different in the short- and long-gradient regimes. In the second case, the expelled electron beam is no longer laminar, and rather has a complex velocity distribution. As we show in the next section, this feature can be attributed to the chaotic character of the electron heating mechanism leading to ejection from the plasma.

Furthermore, these 2D PIC simulations show that a quasistatic magnetic field develops at the plasma surface [see the map of the magnetic field in Fig. 7(c), where a red area indicating a high magnetic field is present close to the plasma surface]. This field grows during the laser-plasma interaction, reaches an amplitude typically of the same order of magnitude as the laser magnetic field, and then persists even after the laser pulse has been reflected by the plasma. A detailed analysis of electron trajectories in these simulations shows that this surface field, which is much larger than the one occurring in the short-gradient regime [compare maps of the magnetic field in Figs. 7(a) and 7(c)], deflects the escaping electrons toward the target surface. This deflection accounts for the fact that the electron angular distributions observed in this regime are essentially centered between the specular direction and target surface. Test simulations have been performed to check that the reflected laser field plays no role in the deflection of electrons after their ejection from the plasma, in strong contrast to what is observed in the Brunel regime.

Such surface quasistatic fields have already been reported in multiple studies of the interaction of intense lasers with dense plasmas (see, e.g., Refs. [62-66]), and can be induced by a variety of physical processes (see, e.g., Refs. [67-70]). In the present case, our simulations indicate that their development can be attributed to the "fountain effect" described in Ref. [70], where they originate from the plasma cold return current that compensates for the lateral charge ejection from the laser focal volume. This is supported by the fact that these fields are not observed at all in plane wave simulations, where the plasma surface is homogeneously illuminated by the laser field.

In contrast to the short-gradient regime, here the spatial properties of the electron beam do not provide much insight into the involved electron heating mechanism. To identify this mechanism, we now turn to a more detailed numerical investigation.

\section{Electron heating mechanism in the long-gradient regime \\ 1. Importance of the reflected laser field}

Our analysis of the electron heating mechanism in the long-gradient regime is based on a set of $2 \mathrm{D}$ plane wave PIC simulations [49] for three different physical configurations. Their key results are summarized in Fig. 8, and shed light on the underlying physical mechanism. The upper panels (case A) correspond to the interaction of an ultraintense laser pulse with a dense plasma in the longgradient regime, i.e., the same physical configuration as in Figs. 7(c)-7(d) and as in our experiment: they display the temporal evolution of the plasma electron density [Fig. 8(a)] and the $x-p_{x}$ phase-space distribution of electrons [Fig. 8(d)] at the time when electron ejection from the plasma is observed to start [blue dashed line in Fig. 8(a)].

The middle panels of Fig. 8 (case B) display the same quantities, but now in a situation where the plasma profile has been truncated for densities $n>0.4 n_{c} \cos ^{2} \theta$, i.e., keeping only the underdense part of the plasma, such that there is hardly any reflection of the incident laser by the plasma. This underdense plasma layer, surrounded by vacuum on both sides, is irradiated by the same laser beam as before, but also by a second beam of slightly lower intensity (80\%), symmetrically arriving from the other side of the plasma. The role of this second laser is to emulate the beam reflected by the dense part of the plasma in case A. The key point here is that both the temporal dynamics of the plasma density profile and the electron phase-space distributions look very similar for cases A and B.

By contrast, if the truncated plasma layer is irradiated by one laser beam only (case C, lower panels of Fig. 8), the plasma dynamics becomes totally different. More specifically, while similar upward electron ejections are observed in cases A (corresponding to the electron signal observed 

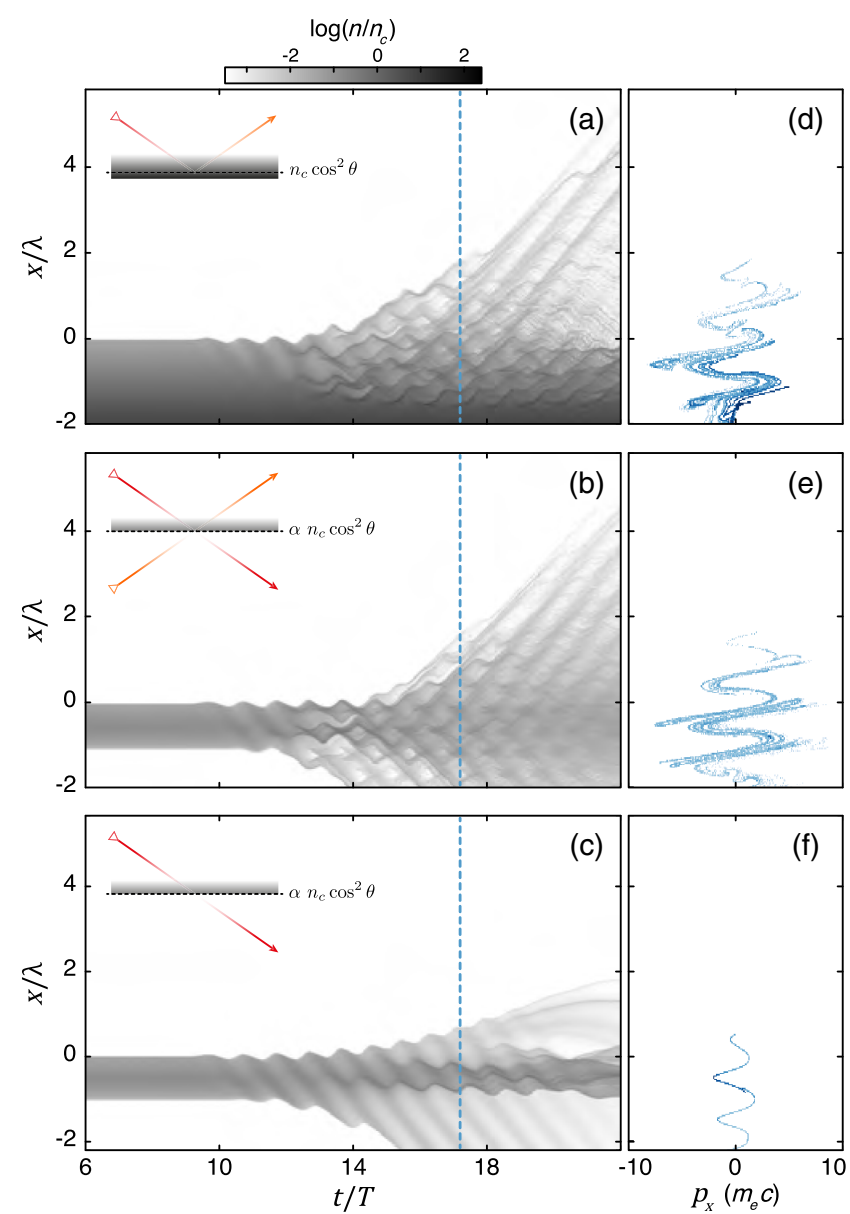

FIG. 8. Set of 2D plane wave PIC simulations carried out to reveal the role of the laser field reflected by the plasma. Each line corresponds to a different physical case [see insets in (a)-(c)]. Case A (upper line) corresponds to an overdense plasma with a density gradient of scale length $L=\lambda$, irradiated by a single laser beam with an incidence angle $\theta_{i}=55^{\circ}$ and $a_{0}=2.5$. In case $\mathrm{B}$, this plasma has been truncated for $n>0.4 n_{c} \cos ^{2} \theta$, keeping only the underdense part of the plasma, which is now irradiated by two almost identical laser beams (same parameters as in case A) arriving symmetrically from both sides of the plasma layer. In case $\mathrm{C}$, this same plasma layer is irradiated only by the upper laser beam. In these three cases, plots of the temporal evolution of the plasma electron density [(a)-(c)] and snapshots of the $x-p_{x}$ phase space distribution of electrons [(d)-(f), taken at the time indicated by the blue line in (a)-(c)] are displayed. The multilayered phase space distributions of (d) and (e) strongly contrast with the smooth regular distribution of (f), and are typical of chaotic dynamics resulting from a repetitive stretching and folding effect in phase space.

in our experiment) and B, this electron emission is strongly reduced in case $\mathrm{C}$ : fewer electrons are emitted, and they have much weaker velocities.

This toy model study leads to two important conclusions: (i) the comparison of cases $\mathrm{A}$ and $\mathrm{B}$ indicates that in the long-gradient regime, the coupling mechanism leading to electron ejection mostly occurs in the underdense part of the density gradient, (ii) the comparison of cases B and C indicates that the overdense part of the plasma nonetheless plays a key role, by producing a reflected beam that, when crossing and interfering with the incident beam, strongly modifies the dynamics of electrons in the underdense plasma layer. As we explain in the next section, the electron heating mechanism coming into play in such a physical situation has already been well identified in the existing literature.

\section{Introduction to stochastic heating}

Let us start by considering a free electron (i.e., without any collective plasma effects involved) exposed to two noncollinear ultraintense laser beams. Using a quantum description of the field as an ensemble of photons provides a simple way to understand that this electron can gain more energy than when exposed to a single laser beam. When a single laser beam (assumed to be a plane wave) is present, it is well known that photon absorption processes are hindered as they do not conserve both energy and momentum of the total system. By contrast, when two noncollinear beams are present, the combined absorption of multiple photons simultaneously from both beams is allowed, because the availability of photons with different $\mathbf{k}$ vectors makes it possible to conserve both energy and momentum of the total system. In other words, the presence of a second beam allows for energy absorption by the electron from the laser field, through a process that can be defined as stimulated multiphoton Compton scattering [71].

For large field amplitudes, the laser field can be treated classically, and many previous studies in the literature have shown that electron dynamics in these combined noncollinear fields is not integrable and gets chaotic for high enough laser amplitudes (typically $a_{0} \gtrsim 0.15$ for at least one of the two beams). This results in large energy gains, and this efficient regime of energy absorption by electrons is known as stochastic heating [34,36,72-78] —although the name chaotic heating would probably be more appropriate here, since the system is perfectly deterministic and involves no stochastic process.

This effect is obviously not restricted to isolated free electrons: it can equally occur for electrons in an underdense plasma, leading to an energy absorption process where neither collisions nor collective plasma effects play any major role. Such a coupling of the plasma with multiple laser beams has been studied experimentally in Ref. [79] by exposing an underdense plasma to two laser beams (like in case B of Fig. 8). It is also known to play a role in electron injection in laser-driven plasma wakefield accelerators by the colliding pulse scheme $[80,81]$.

To the best of our knowledge, Ref. [35] was the first to point out that this mechanism should also come into play when a small underdense plasma layer is present at the surface of a dense plasma exposed to a single laser beam. In this case, the required second noncollinear laser beam 
results from the reflection of the single input beam by the dense plasma. Electrons in the underdense plasma are then exposed to the standing wave formed in front of the dense plasma by the superposition of the incident and reflected beams, and can gain energy by stochastic heating: this is precisely how we interpret our present experimental results in the long-gradient regime.

\section{Numerical evidence for stochastic heating in the long-gradient regime}

With the help of simulations, we now support this interpretation by providing evidence that stochastic heating indeed occurs in the long-gradient regime. To this end, we analyze the electron phase-space distributions in cases A-C [Figs. 8(d)-8(f)] and their temporal evolution during the laser-plasma interaction (see Movie M1 in Supplemental Material [39]). When a single laser beam is present (case C), electrons are observed to simply oscillate nonlinearly in the laser field, leading to a smooth and regular phase space distribution [Fig. 8(f) and Movie M1 [39]). In striking contrast, in cases A and B, electron dynamics in the standing wave resulting from the superposition of two noncollinear laser beams is complex: the key point is that we observe a very strong local "stretching and folding" effect on the phase-space distribution, around each node of the standing wave (see movie M1 [39]).

Such a stretching and folding effect results in very different trajectories for particles that are initially very close in phase space: this is known to be one of the most typical routes to chaotic dynamic [82], exemplified in the well-known horseshoe map models. This repetitive stretching and folding eventually results in a highly structured, multilayered phase-space distribution [Figs. 8(d) and 8(e)], where electrons at a given spatial position have a complex momentum distribution, typical of chaotic dynamics. The striking contrast between these highly structured phasespace distributions and the smooth distribution observed in case C [Fig. 8(f)] again demonstrates the impact of stochastic heating on electron dynamics in the underdense part of the plasma. Furthermore, the comparison of these phase-space distributions with the type of distribution observed for the Brunel mechanism (Fig. 1) is illustrative of the major difference in the dynamics of the system between the short- and long-gradient regimes.

The chaotic character of the electron dynamics can be further supported by the calculation of the Lyapunov exponents of plasma electrons (see Supplemental Material [39]), which should be positive in the case of chaotic dynamics. The Lyapunov exponent $\sigma_{p_{x}}$ for the $p_{x}$ variable, obtained from 2D plane wave PIC simulations of case A of Fig. 8, are displayed in Fig. 9, as a function of the incident laser amplitude. This exponent is negative at low intensity, and gets positive when $a_{0} \gtrsim 0.15$, thus pointing to chaotic dynamics. This threshold in laser intensity is

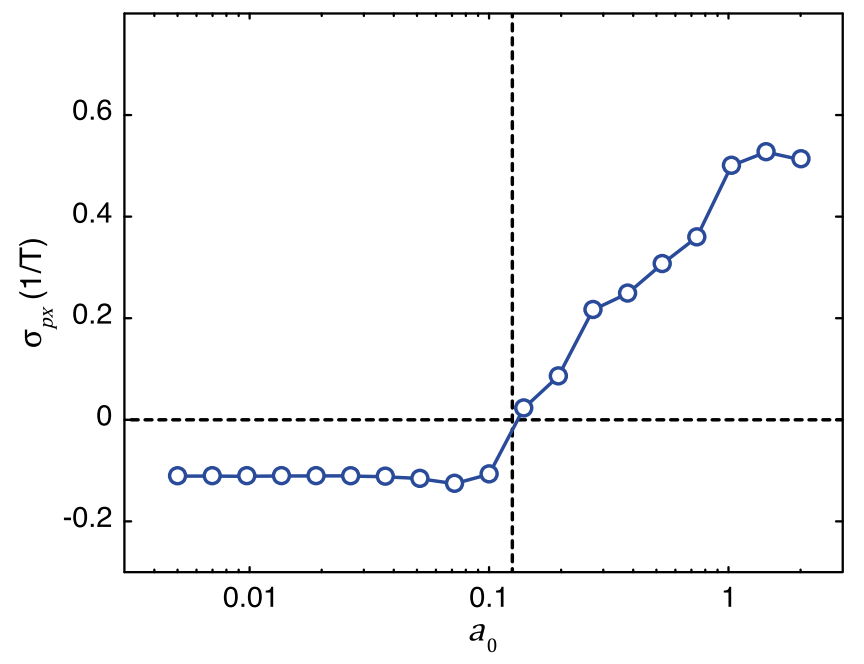

FIG. 9. Lyapunov exponent for the $p_{x}$ variable in the longgradient regime, as a function of $a_{0}$. Simulations have been performed in the physical configuration of case A of Fig. 8. The onset of stochastic heating in the standing wave formed in the underdense part of the plasma by the incident and reflected laser fields is indicated by the fact that the Lyapunov exponent becomes positive. The calculation procedure used to deduce this exponent from the results of 2D plane wave PIC simulations is explained in the Supplemental Material [39].

consistent with early theoretical investigations of stochastic heating $[36,72]$.

\section{Absorption mechanism at lower laser intensities}

Figure 9 shows that for lower laser amplitudes $a_{0} \ll 1$, the Lyapunov exponent $\sigma$ becomes negative, suggesting that stochastic heating vanishes. In this case, when augmenting $L$, we instead observe in simulations a transition from a Brunel-dominated regime to a regime where the well-known resonant absorption mechanism becomes dominant. This is supported by Fig. 10, showing results from 2D plane wave PIC simulations in the long-gradient regime $L=\lambda / 1.5$ for two different laser amplitudes $a_{0}=0.1$ and $a_{0}=3.5$. At a lower laser amplitude, $a_{0}=0.1$, one can clearly observe in Fig. 10(a) a resonant excitation of plasma waves around the critical density $n=n_{c}$. The resonant growth of these plasma waves eventually leads to wave breaking and subsequent ejection of hot electrons after the interaction. The underdense part of the plasma gradient $\left(n<n_{c}\right)$ is only very weakly perturbed in this case.

However, for larger laser amplitudes $a_{0}=3.5$ (as in our experiments), Fig. 10(b) shows that the resonant growth of plasma waves at $n=n_{c}$ vanishes. As opposed to the "classical" case $\left(a_{0} \ll 1\right)$ where all resonant absorption models assume an initially unperturbed plasma density profile, the electron density profile in the relativistic case is highly perturbed and the laser reflects on a sharp electron profile of density $n>n_{c}$. This prevents the growth of any resonant waves near the critical density. Instead, and as 


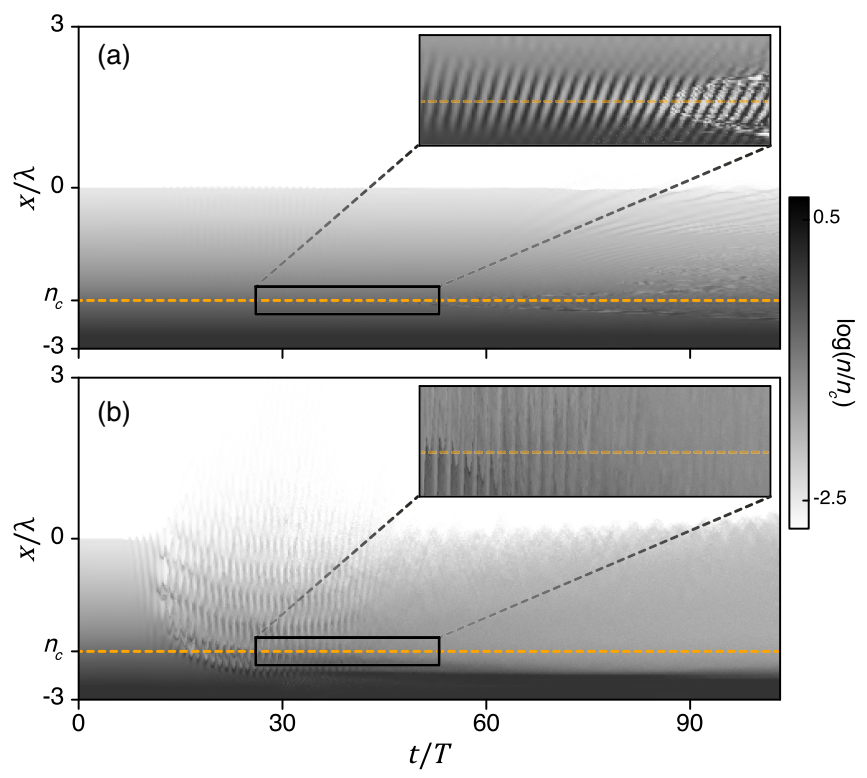

FIG. 10. 2D PIC simulations in the long-gradient regime for two different laser amplitudes. In both cases, the gradient scale length is $L=\lambda / 1.5$ (long-gradient regime) and $\theta_{i}=55^{\circ}$. The gray scale represents the temporal evolution $n_{e}(x, t)$ of the plasma electron density profile along the direction $x$ normal to the plasma surface for (a) $a_{0}=0.1$ and (b) $a_{0}=3.5$. The insets represent enlargements centered around the critical electron density of the initial plasma profile $n_{e}(x, t=0)=n_{c}$ (shown by the orange dashed line).

previously described, the interference pattern produced by the incident and reflected fields within the underdense part of the density gradient is responsible for stochastic heating of electrons, and results in a highly perturbed density profile.

\section{Effect of the laser polarization, plasma reflectivity}

With the support of the previous physical analysis, we finally discuss the experimental observations on the influence of the laser polarization direction (Fig. 4) and the evolution of the plasma reflectivity with $L$, for both $s$ and $p$ polarizations (Fig. 5).

In the short-gradient regime, switching the polarization from $p$ to $s$ is expected to suppress both electron emission and harmonic generation, as observed experimentally [Figs. 4(a) and 4(b)], because the laser $E$-field component normal to the plasma surface is the main driving force of Brunel absorption at the laser intensities considered here. In contrast, when stochastic heating is involved, the polarization direction of the incident laser beam is not expected to have a strong influence, since the plasma surface only comes into play by producing a reflected wave: this explains why, in the long-gradient regime, the electron signal is experimentally observed to still persist in $s$ polarization [Figs. 4(c) and 4(d)]. This experimental observation alone makes a strong case against an interpretation of the long-gradient regime in terms of resonant absorption, which should rather be very sensitive to the laser polarization.

In the long-gradient regime, a spatial degradation of the reflected laser beam wave fronts is observed right after the target in the simulation of Fig. 7(c), while the beam wave front is preserved in the short-gradient regime [Fig. 7(a)]. This is qualitatively consistent with experimental observations (Fig. 5(b)), where the laser beam intensity profile far from the target is observed to become degraded for long gradients. The chaotic character of the electron dynamics, identified in the previous section, affects the laser beam propagation in the underdense plasma layer, and thus provides a possible interpretation for this degradation of the reflected laser wave fronts.

For short density gradients, the reflectivity is lower in $p(\approx 50 \%)$ than in $s$ (close to $100 \%)$ polarization, while similar values $(\approx 70 \%)$ are observed for both polarizations in the long-gradient regime. This is consistent with the results of Fig. 4 on relativistic electron emission, and with our previous interpretation of the interaction: in $s$ polarization, for short $L$, Brunel absorption is suppressed, while for long $L$ stochastic heating is still present.

PIC simulations can be exploited to get even more insight into the redistribution of the initial laser energy after the laser-plasma interaction. The different distributions obtained for both short- and long-gradient regimes, and for both $p$ and $s$ laser polarizations, are displayed as pie charts in Fig. 11. For short gradients and $p$ polarization, around $25 \%$ of the laser is converted into harmonics of the laser frequency (mostly low orders), and about $30 \%$ is deposited as kinetic energy of the plasma particles (including the relativistic electrons observed in our experiment). When the polarization is switched to $s$, these two contributions get considerably reduced, down to around 5\% each, leading to a much higher reflectivity of the fundamental laser frequency. For long gradients, by contrast, very little energy is converted into harmonics, regardless of polarization. As already emphasized, the energy stored into quasistatic fields around the plasma surface significantly increases compared to the short-gradient regime. In $p$ polarization, the fraction of energy going into particle kinetic energy is only slightly weaker than in the shortgradient case, and gets reduced by about $50 \%$ in $s$ polarization. The reflectivity for the fundamental frequency remains similar for both polarizations, while more energy goes into scattered light in $s$ polarization.

From a more quantitative point of view, 2D PIC simulations also properly reproduce the evolution with $L$ of the plasma reflectivity at the fundamental laser frequency, for both $s$ and $p$ polarizations (Fig. 5). Moreover, this evolution is consistent with our previous interpretation. For $p$ polarization, starting from $L \simeq 0$, this reflectivity first decreases, as one approaches the optimum length for the generation of harmonics and relativistic electrons ( $L \simeq \lambda / 15$, see Fig. 3), which of course both occur at 


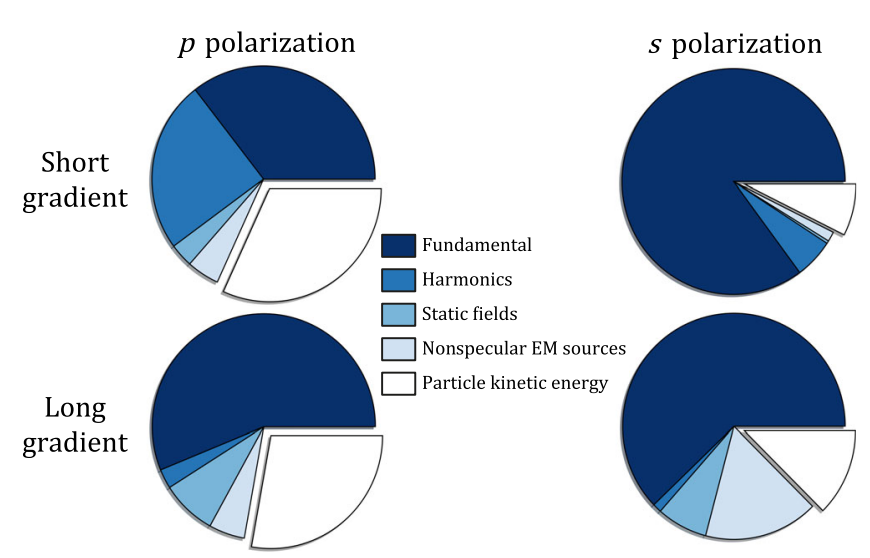

FIG. 11. Distribution of the initial laser pulse energy after the interaction, in different conditions. These pie charts summarize how the laser energy is distributed after the laser-plasma interaction, in the short-gradient (upper line) and long-gradient (lower line) regimes, for $p$ (left-hand column) and $s$ (right-hand column) polarizations. Five categories have been numerically separated, indicated in the legend: electromagnetic (EM) energy in the fundamental laser frequency in the specular direction, in the harmonics of the laser frequency in the specular direction, in quasistatic fields (i.e., with a frequency lower than the laser frequency), in nonstatic fields in the nonspecular direction, and kinetic energy of plasma particles. The results are obtained from 2D PIC simulations with $a_{0}=3, \theta_{i}=55^{\circ}$, and $L_{1}=\lambda / 15$ for the short-gradient regime and $L_{2}=\lambda / 1.5$ in the long-gradient regime.

the expense of laser energy (see Fig. 11). Beyond this point, the energy converted to particle kinetic energy does not vary much, but the one converted to harmonics progressively drops to 0 : this explains why the reflectivity then gradually increases beyond $L \simeq 0.1 \lambda$.

\section{E. Conclusion of the physical analysis}

The previous combination of multiple experimental observables and PIC simulations has provided strong evidence for the transition from Brunel absorption to stochastic heating as the density gradient scale length $L$ is increased, while no evidence of resonance absorption has been observed in this ultrahigh laser intensity regime.

The intuitive physical insight underlying this transition is that the Brunel mechanism requires a sharp interface, such that the amplitude of the quivering motion of electrons in the laser field exceeds the length scale of this interface. On the contrary, stochastic heating can only occur if electrons are present within the standing wave interference pattern formed by the incident and reflected fields: it will therefore be favored by longer density gradients, for which this interference zone contains a larger number of particles provided by the underdense part of the plasma located just in front of the laser reflection point.

It however remains difficult to predict analytically the value $L_{t}$ of the density gradient scale length for which this transition occurs. In the next section, we determine this transition length experimentally as a function of the laser incidence angle on target $\theta_{i}$ - an essential physical parameter, since it affects the point of the density gradient where laser reflection occurs. We also discuss the effect of this angle on the properties of the emitted electron beams.

\section{EFFECT OF THE LASER INCIDENCE ANGLE}

\section{A. Evolution of the transition gradient scale length}

We have repeated the previous measurements, carried out for $\theta_{i}=55^{\circ}$, for five other incidence angles $\theta_{i}$ ranging from $40^{\circ}$ to $65^{\circ}$. For each angle, the density gradient scale length was systematically varied by changing the prepulse delay, and was measured using the SDI technique. The main outcomes of this experiment are summarized in Fig. 12. The left-hand panels show how the electron beam angular profile in the incidence plane evolves as a function of $L$, for three different incidence angles. In all cases, the same type of transition as reported in Figs. 2 and 3 is observed, and it occurs for shorter values of $L$ as the incidence angle is increased. As before, we observe that this transition is correlated with major changes in the electron angle-energy distribution and in the harmonic emission. The experimental transition length $L_{t}^{e}$ deduced from all these data is plotted in Fig. 12(b) (blue dots), and clearly decreases with $\theta_{i}$.

To get some qualitative insight into this angular dependance, we consider the starting point of Brunel's model [11], which is that the physics of the laser-plasma coupling changes when the quivering amplitude $\Delta z_{e}$ of plasma electrons starts exceeding the typical spatial extent $d$ of the plasma-vacuum interface. We then define-somewhat arbitrarily - this spatial extent as $d=\left|z_{s}-z_{c}\right|$, the distance between the effective reflective surface of the plasma, located at $z_{s}\left(\theta_{i}\right)$, such that $n\left(z_{s}\right)=n_{c} \cos ^{2} \theta_{i}$, and the fixed point corresponding to the location of the critical plasma density $z_{c}$, such that $n\left(z_{c}\right)=n_{c}$ [see sketch in Fig. 12(b)]. $d$ can be calculated by using the standard assumption of an exponential density profile at the target surface [83]: $n(z)=n_{0} \exp (-z / L)$, with $n_{0}$ the maximum plasma density in the target, reached at $z=0$. This leads to $d=-2 L \ln \left(\cos \theta_{i}\right)$.

In the simplest possible approach, the electron quivering amplitude can be considered to be the one of free electrons in the laser field, which is $\Delta z_{e} \approx \lambda a_{0}^{2} / 2 \pi\left(a_{0}^{2}+1\right)$. At sufficiently high intensity, well into the relativistic regime $\left(a_{0} \gg 1\right), \Delta z_{e} \approx \lambda / 2 \pi$ is quasi-independent of the laser field amplitude. The condition $\Delta z_{e}=d\left(\theta_{i}\right)$ then leads to a simple theoretical expression for the transition gradient scale length:

$$
\frac{L_{t}}{\lambda}=-\frac{1}{4 \pi \ln \left(\cos \theta_{i}\right)} .
$$

This simple analysis indeed predicts an angular dependence of $L_{t}$ on $\theta_{i}$ : physically, this is because for a given plasma 

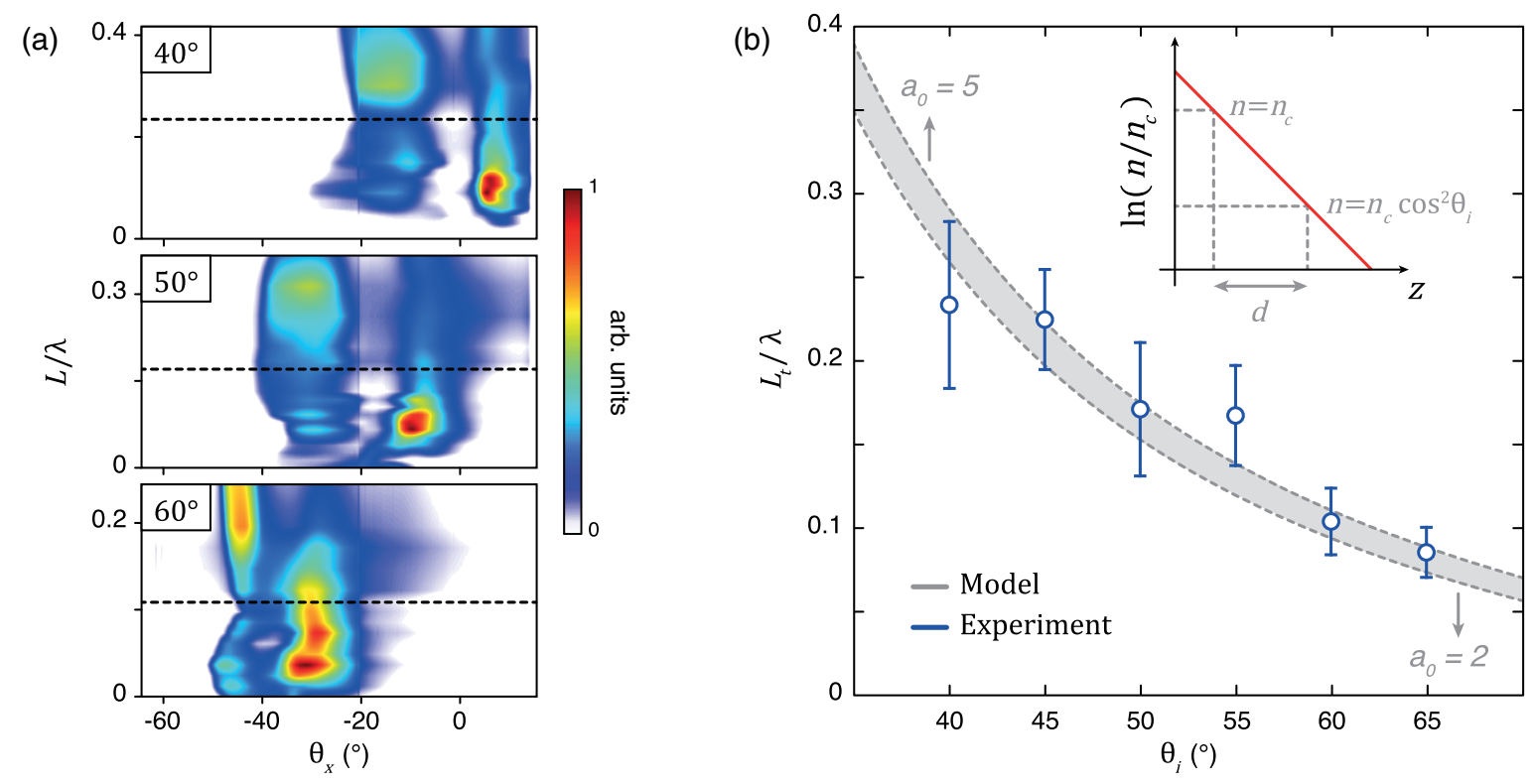

FIG. 12. Effect of the laser incidence angle on the laser-plasma coupling. Panel (a) shows the evolutions with $L$ of the high-energy electron beam angular profile in the incidence plane, measured for three different incidence angles, for a laser field amplitude $a_{0} \approx 3.5$. For each angle, $L$ is measured experimentally using the SDI technique. The value $L_{t}^{e}$ where a transition occurs is clearly observed to decrease with $\theta_{i}$. The measured evolution of $L_{t}^{e}$ with $\theta_{i}$ is plotted in (b), where it is compared to the simple model described in the text. The predictions of this model are shown for a range of laser amplitudes $2 \leq a_{0} \leq 5$, to account for the experimental uncertainty on this amplitude. These predictions are very close to the high-amplitude limit given by Eq. (1).

density profile at the surface (i.e., a fixed $L$ ), the distance $d$ increases with $\theta_{i}$, due to the well-known angular dependence of the effective critical density, $n_{s}=n_{c} \cos ^{2} \theta_{i}$. Figure 12(b) displays a quantitative comparison of the experimental values $L_{t}^{e}$ with the prediction $L_{t}$ of this model, showing a good agreement. Despite its extreme simplicity, this model can still account for the angular dependence observed experimentally. Yet it becomes inappropriate for small angles - a regime that could not be investigated in our experiment for practical reasons. Indeed, as $\theta_{i} \rightarrow 0$, $n_{c} \cos ^{2} \theta_{i} \rightarrow n_{c}$, and hence, the physical distance between these two densities tends to 0 , whatever the value of the density gradient $L$. Therefore, this model leads to the physically unsatisfactory prediction that, at incidences close to normal, the Brunel mechanism should dominate even for very large $L$. This is an indication of the limitations of this very basic model, which we only use here to qualitatively explain the trend of the angular dependence observed at large enough incidence angles.

\section{B. Evolution of the electron number and energy}

Several other effects are observed when the incidence angle is changed, depending on the coupling mechanism. This is summarized in Fig. 13, where the evolutions of the electron signal and average energy are displayed as a function of the gradient scale length $L$ and incidence angle $\theta_{i}$, over a range that covers the short- and long-gradient interaction regimes described before. In these plots, the areas associated to these two regimes are separated by a white zone, which corresponds to the transition curve predicted by the simple model of the previous section, and displayed in Fig. 12(b). In the following, we describe these evolutions and suggest tentative interpretations, which will need further investigation to be validated in detail.

Both in the Brunel absorption and stochastic heating regimes, the number of emitted electrons grows with $\theta_{i}$ [see Fig. 13(a)] in the angular range investigated here. This might be attributed to the fact that when $\theta_{i}$ is increased, the target area covered by the laser focal spots increases, so that more electrons get involved in the interaction.

As far as energy is concerned, it is hardly affected by the gradient scale length $L$ in each interaction regime, while the effect of incidence angle is different for the these two regimes. In the Brunel regime, the electron average energy only weakly changes with incidence angle, slightly decreasing for larger angles. Physically, in this regime, most of the electron energy gain is due to the VLA process, which is not expected to depend on the incidence angle since it occurs in vacuum. Changing $\theta_{i}$ might, however, modify the conditions of injection of electrons in the reflected field (e.g., their initial energy), and this might explain the observed slight angular dependence of the energy of VLA electrons.

By contrast, in the stochastic heating regime, the electron spectrum clearly shifts to higher energies as $\theta_{i}$ is increased [see Fig. 13(b)], up to about $20 \mathrm{MeV}$. This might be due to 

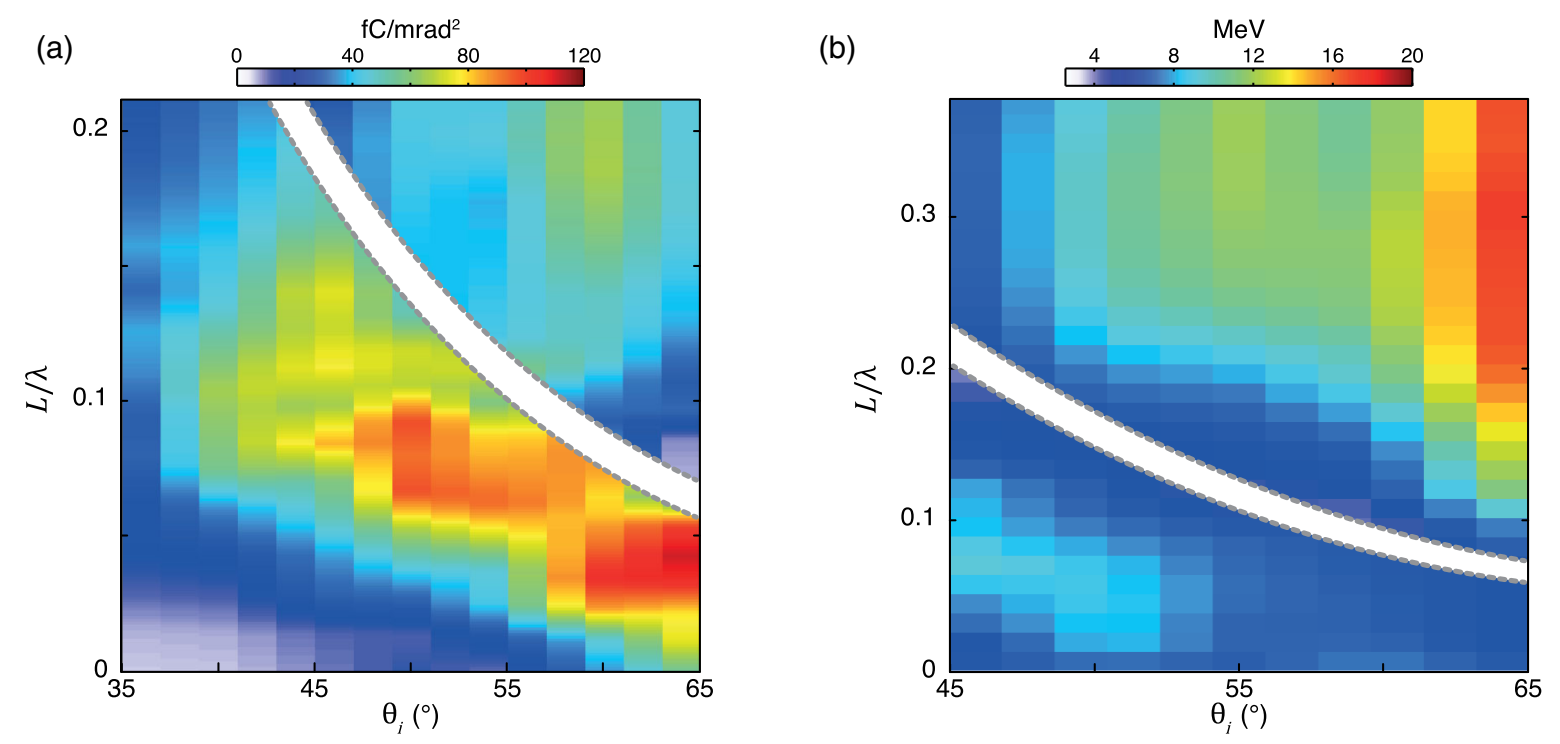

FIG. 13. Measured electron beam properties as a function of key interaction parameters. Panel (a) shows the experimental evolution of the number of emitted high-energy electrons $(E>1 \mathrm{MeV})$ as a function of the incidence angle $\theta_{i}$ and density gradient scale length $L$, for $a_{0}=3.5$ and $p$ polarization of the laser. The white area corresponds to the transition between the Brunel and stochastic heating coupling regimes, identified in Fig. 12. Below this border, the displayed signal corresponds to the integration of the electron beam spatial profile on the right of the ponderomotive hole (i.e., VLA electrons only). Above this border, it corresponds to the integration of the electron beam spatial profile on the left of this hole only. Panel (b) shows the average electron energy $\left[E_{m}=\int_{E>3 \mathrm{MeV}} E n(E) d E / \int n(E) d E\right]$ as a function of the same physical parameters. Here again, the electron spectra used for this calculation were selected on the right of the ponderomotive hole for short gradients, and on its left for long gradients.

the fact that, all laser parameters being kept fixed, the "lifetime" of the interference pattern formed by the incident and reflected fields increases with $\theta_{i}$. A simple analytical calculation indeed gives the following equation for the duration $\tau_{i}$ of this standing wave:

$$
\tau_{i}=\sin \theta_{i}\left(\tau_{L}+\frac{w}{c} \tan \theta_{i}\right),
$$

where $\tau_{L}$ is the laser pulse duration and $w$ the laser focal spot size. As a result, for larger $\theta_{i}$, the stochastic heating process driven by the interference pattern can last longer, leading to a larger final energy gain for electrons, which could account for the experimental observation of Fig. 13.

\section{CONCLUSION}

In conclusion, we have combined state-of-the-art experiments and PIC simulations to provide the first unambiguous experimental evidence of the transition from Brunel absorption to a different laser-plasma coupling mechanism, as the steepness of the plasma surface is varied. This mechanism has been identified to be stochastic heating of electrons in the underdense plasma layer at the target surface, driven by the standing wave formed by the incident and reflected laser waves. This work has enabled the identification of clear signatures of these two coupling mechanisms, carried by the relativistic electron emission towards vacuum, the generated harmonic signal, and even the spatial profile of the reflected laser beam. At the laser intensities considered here, no evidence of the process known as resonant absorption has been found.

These signatures should prove extremely useful for the interpretation of a broad range of topical experiments performed with high-power ultrashort lasers, related, e.g., to ion and electron acceleration, or to the generation of short-wavelength light and attosecond pulses. In the latter case, ultrahigh contrast pulses are required, generally obtained with plasma mirrors or by frequency doubling after the final compression stage, and our results confirm that such experiments involve the Brunel mechanism. But in many other experiments-e.g., for ion acceleration from dense plasmas-preserving very steep interfaces is not strictly necessary. Such experiments are therefore often performed without contrast improvement after temporal compression: the laser pulses then typically have a very high temporal contrast up to a few picoseconds before the main pulse, but their short-time contrast is often not as good, due, e.g., to remaining high-order terms in the pulse spectral phase. With typical plasma expansion velocities in the $50 \mathrm{~nm} / \mathrm{ps}$ range, this picosecond pedestal will lead to density gradient scale lengths of the order of $\lambda$ at the arrival of the main pulse. In these conditions, stochastic heating should be the dominant coupling mechanism, and can now be readily identified through the multiple signatures described in this work. 


\section{ACKNOWLEDGMENTS}

We are grateful to F. Réau, C. Pothier and D. Garzella for operating the UHI100 laser source. The research leading to this work has been funded by the ERC (Grant ExCoMet, No. 694596), the Agence Nationale pour la Recherche under Contract No. ANR-14-CE32-0011-03 APERO, and LASERLAB-EUROPE (Grant Agreement No. 654148, EC's H2020 Framework Programme). An award of computer time (PICSSAR-INCITE) was provided by the Innovative and Novel Computational Impact on Theory and Experiment (INCITE) program. This research used resources of the Argonne Leadership Computing Facility (MIRA,THETA), which is a U.S. DOE Office of Science User Facility supported under Contract No. DE-AC0206CH11357 as well as resources (CORI) of the National Energy Research Scientific Computing Center, a U.S. DOE Office of Science User Facility supported by the Office of Science of the U.S. Department of Energy under Contract No. DE-AC02-05CH11231.

[1] S. Atzeni and J. Meyer-ter vehn, The Physics of Inertial Fusion (Clarendon, Oxford, 2004).

[2] B. A. Remington, R. P. Drake, H. Takabe, and D. Arnett, A Review of Astrophysics Experiments on Intense Lasers, Phys. Plasmas 7, 1641 (2000).

[3] H. Daido, M. Nishiuchi, and A. S. Pirozhkov, Review of Laser-Driven Ion Sources and Their Applications, Rep. Prog. Phys. 75, 056401 (2012).

[4] A. Macchi, M. Borghesi, and M. Passoni, Ion Acceleration by Superintense Laser-Plasma Interaction, Rev. Mod. Phys. 85, 751 (2013).

[5] W. L. Kruer, The Physics of Laser Plasma Interactions (Addison-Wesley, Reading, 1988).

[6] C. Garban-Labaune, E. Fabre, C. E. Max, R. Fabbro, F. Amiranoff, J. Virmont, M. Weinfeld, and A. Michard, Effect of Laser Wavelength and Pulse Duration on Laser-Light Absorption and Back Reflection, Phys. Rev. Lett. 48, 1018 (1982).

[7] J. P. Freidberg, R. W. Mitchell, R. L. Morse, and L. I. Rudsinski, Resonant Absorption of Laser Light by Plasma Targets, Phys. Rev. Lett. 28, 795 (1972).

[8] D. W. Forslund, J. M. Kindel, K. Lee, E. L. Lindman, and R. L. Morse, Theory and Simulation of Resonant Absorption in a Hot Plasma, Phys. Rev. A 11, 679 (1975).

[9] K. G. Estabrook, E. J. Valeo, and W. L. Kruer, Two Dimensional Relativistic Simulations of Resonance Absorption, Phys. Fluids 18, 1151 (1975).

[10] D. W. Forslund, J. M. Kindel, and K. Lee, Theory of Hot Electron Spectra at High Laser Intensity, Phys. Rev. Lett. 39, 284 (1977).

[11] F. Brunel, Not So Resonant, Resonant Absorption, Phys. Rev. Lett. 59, 52 (1987).

[12] P. B. Corkum, Plasma Perspective on Strong Field Multiphoton Ionization, Phys. Rev. Lett. 71, 1994 (1993).

[13] M. Nantel, J. Itatani, A. C. Tien, J. Faure, D. Kaplan, M. Bauvier, T. Buma, P. Van Rompay, J. Nee, P. P. Pronko,
D. Umstadter, and G. A. Mourou, Temporal Contrast in Ti: Sapphire Lasers, Characterization and Control, IEEE J. Sel. Top. Quantum Electron. 4, 449 (1998).

[14] J. Itatani, J. Faure, M. Nantel, G. Mourou, and S. Watanabe, Suppression of the Amplified Spontaneous Emission in Chirped-Pulse-Amplification Lasers by Clean High-Energy Seed-Pulse Injection, Opt. Commun. 148, 70 (1998).

[15] H. C. Kapteyn, M. M. Murnane, A. Szoke, and R. W. Falcone, Prepulse Energy Suppression for High-Energy Ultrashort Pulses Using Self-Induced Plasma Shuttering, Opt. Lett. 16, 490 (1991).

[16] G. Doumy, F. Quere, O. Gobert, M. Perdrix, P. Martin, P. Audebert, J. C. Gauthier, J.-P. Geindre, and T. Wittmann, Complete Characterization of a Plasma Mirror for the Production of High Contrast Ultraintense Laser Pulses, Phys. Rev. E 69, 026402 (2004).

[17] B. Dromey, S. Kar, M. Zepf, and P. Foster, The Plasma Mirror-A Subpicosecond Optical Switch for Ultrahigh Power Lasers, Rev. Sci. Instrum. 75, 645 (2004).

[18] A. Lévy, T. Ceccotti, P. Dé Oliveira, F. Réau, M. Perdrix, F. Quéré, P. Monot, M. Bougeard, H. Lagadec, P. Martin, P. Audebert, and J. P. Geindre, Double Plasma Mirror for Ultrahigh Temporal Contrast Ultraintense, Opt. Lett. 32, 310 (2007).

[19] C. Thaury, F. Quéré, J. P. Geindre, A. Lévy, T. Ceccotti, P. Monot, M. Bougeard, F. Réau, P. D’Oliveira, P. Audebert, R. Marjoribanks, and P. Martin, Plasma Mirrors for Ultrahigh Intensity Optics, Nat. Phys. 3, 424 (2007).

[20] P. Gibbon and A. R. Bell, Collisionless Absorption in Sharp Edged Plasmas, Phys. Rev. Lett. 68, 1535 (1992).

[21] P. Gibbon, A. Andreev, E. Lefebvre, G. Bonnaud, H. Ruhl, J. Delettrez, and A. R. Bell, Calibration of One Dimensional Boosted Kinetic Codes for Modeling High Intensity Laser Solid Interactions, Phys. Plasmas 6, 947 (1999).

[22] C. Thaury and F. Quéré, High Order Harmonic and Attosecond Pulse Generation on Plasma Mirrors: Basic Mechanisms, J. Phys. B. 43, 213001 (2010).

[23] F. N. Beg, A. R. Bell, A. E. Dangor, C. N. Danson, A. P. Fews, M. E. Glinsky, B. A. Hammel, P. Lee, P. A. Norreys, and M. Tatarakis, A Study of Picosecond Laser Solid Interactions Up to $10^{19} \mathrm{~W} / \mathrm{cm}^{2}$, Phys. Plasmas 4, 447 (1997).

[24] S. C. Wilks, A. B. Langdon, T. E. Cowan, M. Roth, M. Singh, S. Hatchett, M. H. Key, D. Pennington, A. MacKinnon, and R. A. Snavely, Energetic Proton Generation in Ultra Intense Laser Solid Interactions, Phys. Plasmas 8, 542 (2001).

[25] K. B. Wharton, S. P. Hatchett, S. C. Wilks, M. H. Key, J. D. Moody, V. Yanovsky, A. A. Offenberger, B. A. Hammel, M. D. Perry, and C. Joshi, Experimental Measurements of Hot Electrons Generated by Ultraintense $\left(10^{19} \mathrm{~W} / \mathrm{cm}^{2}\right)$ Laser Plasma Interactions on Solid Density Targets, Phys. Rev. Lett. 81, 822 (1998).

[26] S. Bastiani, P. Audebert, J. P. Geindre, T. Schlegel, J. C. Gauthier, C. Quoix, G. Hamoniaux, G. Grillon, and A. Antonetti, Hot Electron Distribution Functions in a Subpicosecond Laser Interaction with Solid Targets of Varying Initial Gradient Scale Lengths, Phys. Rev. E 60, 3439 (1999).

[27] D. F. Cai, Y. Q. Gu, Z. J. Zheng, T. S. Wen, S. T. Chunyu, Z. B. Wang, and X.D. Yang, Experimental Study for 
Angular Distribution of the Hot Electrons Generated by Femtosecond Laser Interaction with Solid Targets, Phys. Plasmas 10, 3265 (2003).

[28] A. G. Mordovanakis, J. Easter, N. Naumova, K. Popov, P.-E. Masson-Laborde, B. Hou, I. Sokolov, G. Mourou, I. V. Glazyrin, W. Rozmus et al., Quasimonoenergetic Electron Beams with Relativistic Energies and Ultrashort Duration from Laser Solid Interactions at $0.5 \mathrm{kHz}$, Phys. Rev. Lett. 103, 235001 (2009).

[29] F. Brandl, B. Hidding, J. Osterholz, D. Hemmers, A. Karmakar, A. Pukhov, and G. Pretzler, Directed Acceleration of Electrons from a Solid Surface by Sub-10-fs Laser Pulses, Phys. Rev. Lett. 102, 195001 (2009).

[30] W. Wang, J. Liu, Y. Cai, C. Wang, L. Liu, C. Xia, A. Deng, Y. Xu, Y. Leng, R. Li et al., Angular and Energy Distribution of Fast Electrons Emitted from a Solid Surface Irradiated by Femtosecond Laser Pulses in Various Conditions, Phys. Plasmas 17, 023108 (2010).

[31] Y. Tian, J. Liu, W. Wang, C. Wang, A. Deng, C. Xia, W. Li, L. Cao, H. Lu, H. Zhang et al., Electron Emission at Locked Phases from the Laser-Driven Surface Plasma Wave, Phys. Rev. Lett. 109, 115002 (2012).

[32] M. Thévenet, A. Leblanc, S. Kahaly, H. Vincenti, A. Vernier, F. Quéré, and J. Faure, Vacuum Laser Acceleration of Relativistic Electrons Using Plasma Mirror Injectors, Nat. Phys. 12, 355 (2016).

[33] U. Teubner and P. Gibbon, High-Order Harmonics from Laser-Irradiated Plasma Surfaces, Rev. Mod. Phys. 81, 445 (2009).

[34] J. T. Mendonca and F. Doveil, Stochasticity in Plasmas with Electromagnetic Waves, J. Plasma Phys. 28, 485 (1982).

[35] Y. Sentoku, V. Y. Bychenkov, K. Flippo, A. Maksimchuk, K. Mima, G. Mourou, Z. M. Sheng, and D. Umstadter, High Energy Ion Generation in Interaction of Short Laser Pulse with High Density Plasma, Appl. Phys. B 74, 207 (2002).

[36] Z.-M. Sheng, K. Mima, Y. Sentoku, M. S. Jovanovic, T. Taguchi, J. Zhang, and J. Meyer-ter-Vehn, Stochastic Heating and Acceleration of Electrons in Colliding Laser Fields in Plasma, Phys. Rev. Lett. 88, 055004 (2002).

[37] S. Kahaly, S. Monchocé, H. Vincenti, T. Dzelzainis, B. Dromey, M. Zepf, P. Martin, and F. Quéré, Direct Observation of Density Gradient Effects in Harmonic Generation from Plasma Mirrors, Phys. Rev. Lett. 110, 175001 (2013).

[38] M. Bocoum, F. Böhle, A. Vernier, A. Jullien, J. Faure, and R. Lopez-Martens, Spatial-Domain Interferometer for Measuring Plasma Mirror Expansion, Opt. Lett. 40, 3009 (2015).

[39] See Supplemental Material at http://link.aps.org/ supplemental/10.1103/PhysRevX.9.011050 for a document with additional information on the simulations and experiments as well as a movie of PIC simulation results.

[40] http://blast.lbl.gov/blast-codes-warp.

[41] https://picsar.net.

[42] J.-L. Vay, R. Lehe, H. Vincenti, B. B. Godfrey, I. Haber, and P. Lee, Recent Advances in High-Performance Modeling of Plasma-Based Acceleration Using the Full PIC Method, Nucl. Instrum. Methods Phys. Res., Sect. A 829, 353 (2016).

[43] H. Vincenti and J.-L. Vay, Detailed Analysis of the Effects of Stencil Spatial Variations with Arbitrary High-Order
Finite-Difference Maxwell Solver, Comput. Phys. Commun. 200, 147 (2016).

[44] H. Vincenti, M. Lobet, R. Lehe, R. Sasanka, and J.-L. Vay, An Efficient and Portable SIMD Algorithm for Chargel Current Deposition in Particle-in-Cell Codes, Comput. Phys. Commun. 210, 145 (2017).

[45] H. Vincenti and J.-L. Vay, Ultrahigh-Order Maxwell Solver with Extreme Scalability for Electromagnetic PIC Simulations of Plasmas, Comput. Phys. Commun. 228, 22 (2018).

[46] G. Blaclard, H. Vincenti, R. Lehe, and J. L. Vay, Pseudospectral Maxwell Solvers for an Accurate Modeling of Doppler Harmonic Generation on Plasma Mirrors with Particle in Cell Codes, Phys. Rev. E 96, 033305 (2017).

[47] https://www.alcf.anl.gov/mira.

[48] http://www.nersc.gov/users/computational-systems/cori.

[49] When the incident laser beam has a finite focal spot size, these simulations are performed in the laboratory frame. When the incident laser beam is considered to be a plane wave (infinite focal spot size), the simulation is performed in a Lorentz-boosted frame, such that the laser impinges the plasma at normal incidence. Then, only one spatial coordinate (along the target normal) is taken into account in the simulation, but the three velocity components of particles are calculated. We refer to this second type of simulation as "2D plane wave."

[50] R. Lichters, J. Meyer-ter-Vehn, and A. Pukhov, Short Pulse Laser Harmonics from Oscillating Plasma Surfaces Driven at Relativistic Intensity, Phys. Plasmas 3, 3425 (1996).

[51] B. Dromey, M. Zepf, A. Gopal, K. Lancaster, M. S. Wei, K. Krushelnick, M. Tatarakis, N. Vakakis, S. Moustaizis, R. Kodama, M. Tampo, C. Stoeckl, R. Clarke, H. Habara, D. Neely, S. Karsch, and P. Norreys, High Harmonic Generation in the Relativistic Limit, Nat. Phys. 2, 456 (2006).

[52] S. Gordienko, A. Pukhov, O. Shorokhov, and T. Baeva, Relativistic Doppler Effect: Universal Spectra and Zeptosecond Pulses, Phys. Rev. Lett. 93, 115002 (2004).

[53] T. Baeva, S. Gordienko, and A. Pukhov, Theory of High-Order Harmonic Generation in Relativistic Laser Interaction with Overdense Plasma, Phys. Rev. E 74, 046404 (2006).

[54] D. An der Brugge and A. Pukhov, Enhanced Relativistic Harmonics by Electron Nanobunching, Phys. Plasmas 17, 033110 (2010).

[55] A. A. Gonoskov, A. V. Korzhimanov, A. V. Kim, M. Marklund, and A. M. Sergeev, Ultrarelativistic Nanoplasmonics as a Route towards Extreme-Intensity Attosecond Pulses, Phys. Rev. E 84, 046403 (2011).

[56] A. Debayle, J. Sanz, and L. Gremillet, Self-Consistent Theory of High-Order Harmonic Generation by Relativistic Plasma Mirror, Phys. Rev. E 92, 053108 (2015).

[57] J. Sanz, A. Debayle, and K. Mima, Model for Ultraintense Laser-Plasma Interaction at Normal Incidence, Phys. Rev. E 85, 046411 (2012).

[58] M. Cherednychek and A. Pukhov, Analytical Description of Attosecond Pulse Generation on a Plasma Surface Irradiated by High-Intense Laser Pulses, Quantum Electron. 46, 353 (2016).

[59] G. Malka, E. Lefebvre, and J. L. Miquel, Experimental Observation of Electrons Accelerated in Vacuum to 
Relativistic Energies by a High-Intensity Laser, Phys. Rev. Lett. 78, 3314 (1997).

[60] B. Quesnel and P. Mora, Theory and Simulation of the Interaction of Ultraintense Laser Pulses with Electrons in Vacuum, Phys. Rev. E 58, 3719 (1998).

[61] M. Thévenet, H. Vincenti, and J. Faure, On the Physics of Electron Ejection from Laser-Irradiated Overdense Plasmas, Phys. Plasmas 23, 063119 (2016).

[62] M. Tatarakis, A. Gopal, I. Watts, F. N. Beg, A. E. Dangor, K. Krushelnick, U. Wagner, P. A. Norreys, E. L. Clark, M. Zepf, and R. G. Evans, Measurements of Ultrastrong Magnetic Fields during Relativistic Laser-Plasma Interactions, Phys. Plasmas 9, 2244 (2002).

[63] H. Ruhl, Y. Sentoku, K. Mima, K. A. Tanaka, and R. Kodama, Collimated Electron Jets by Intense LaserBeam-Plasma Surface Interaction under Oblique Incidence, Phys. Rev. Lett. 82, 743 (1999).

[64] Y. T. Li, X. H. Yuan, M. H. Xu, Z. Y. Zheng, Z. M. Sheng, M. Chen, Y. Y. Ma, W. X. Liang, Q. Z. Yu, Y. Zhang et al., Observation of a Fast Electron Beam Emitted along the Surface of a Target Irradiated by Intense Femtosecond Laser Pulses, Phys. Rev. Lett. 96, 165003 (2006).

[65] M. Nakatsutsumi, Y. Sentoku, A. Korzhimanov, S. N. Chen, S. Buffechoux, A. Kon, B. Atherton, P. Audebert, M. Geissel, L. Hurd et al., Self-Generated Surface Magnetic Fields Inhibit Laser-Driven Sheath Acceleration of HighEnergy Protons, Nat. Commun. 9, 280 (2018).

[66] F. Pérez, A. J. Kemp, L. Divol, C. D. Chen, and P. K. Patel, Deflection of MeV Electrons by Self-Generated Magnetic Fields in Intense Laser-Solid Interactions, Phys. Rev. Lett. 111, 245001 (2013).

[67] R. Fabbro and P. Mora, Hot Electrons Behavior in LaserPlane Target Experiments, Phys. Lett. A 90, 48 (1982).

[68] C. Thaury, P. Mora, A. Héron, J.-C. Adam, and T. M. Antonsen, Influence of the Weibel Instability on the Expansion of a Plasma Slab into a Vacuum, Phys. Rev. E 82, 026408 (2010).

[69] A. Kumar, C. Shukla, A. Das, and P. Kaw, Energy Principle for Excitations in Plasmas with Counterstreaming Electron Flows, AIP Adv. 8, 055213 (2018).

[70] R. N. Sudan, Mechanism for the Generation of $10^{9} \mathrm{G}$ Magnetic Fields in the Interaction of Ultraintense Short Laser Pulse with an Overdense Plasma Target, Phys. Rev. Lett. 70, 3075 (1993).
[71] D. D. Meyerhofer, High-Intensity-Laser-Electron Scattering, IEEE J. Quantum Electron. 33, 1935 (1997).

[72] J. T. Mendonca, Threshold for Electron Heating by Two Electromagnetic Waves, Phys. Rev. A 28, 3592 (1983).

[73] J.-M. Rax, Compton Harmonic Resonances, Stochastic Instabilities, Quasilinear Diffusion, and Collisionless Damping with Ultra-High-Intensity Laser Waves, Phys. Fluids B 4, 3962 (1992).

[74] D. Patin, E. Lefebvre, A. Bourdier, and E. D'humieres, Stochastic Heating in Ultra High Intensity Laser-Plasma Interaction: Theory and PIC Code Simulations, Laser Part. Beams 24, 223 (2006).

[75] A. Bourdier, D. Patin, and E. Lefebvre, Stochastic Heating in Ultra High Intensity Laser-Plasma Interaction, Physica D (Amsterdam) 206D, 1 (2005).

[76] A. G. Krygier, D. W. Schumacher, and R. R. Freeman, On the Origin of Super-Hot Electrons from Intense Laser Interactions with Solid Targets Having Moderate Scale Length Preformed Plasmas, Phys. Plasmas 21, 023112 (2014).

[77] B. S. Paradkar, S. I. Krasheninnikov, and F. N. Beg, Mechanism of Heating of Pre-Formed Plasma Electrons in Relativistic Laser-Matter Interaction, Phys. Plasmas 19, 060703 (2012).

[78] A. J. Kemp, Y. Sentoku, and M. Tabak, Hot-Electron Energy Coupling in Ultraintense Laser-Matter Interaction, Phys. Rev. E 79, 066406 (2009).

[79] P. Zhang, N. Saleh, S. Chen, Z. M. Sheng, and D. Umstadter, Laser-Energy Transfer and Enhancement of Plasma Waves and Electron Beams by Interfering High-Intensity Laser Pulses, Phys. Rev. Lett. 91, 225001 (2003).

[80] J. Faure, C. Rechatin, A. Norlin, A. Lifschitz, Y. Glinec, and V. Malka, Controlled Injection and Acceleration of Electrons in Plasma Wakefields by Colliding Laser Pulses, Nature (London) 444, 737 (2006).

[81] S. Rassou, A. Bourdier, and M. Drouin, Role of Stochastic Heating in Wakefield Acceleration when Optical Injection Is Used, Phys. Plasmas 21, 083101 (2014).

[82] S. H. Strogatz, Nonlinear Dynamics and Chaos: With Applications to Physics, Biology, Chemistry, and Engineering, 2nd ed. (CRC Press, Boca Raton, FL, 2018).

[83] Ya. B. Zel'dovich and Yu. P. Raizer, Physics of Shock Waves and High-Temperature Hydrodynamic Phenomena (Dover Publications, New York, 2002). 